\title{
POLITICAS POBLACIONALES EN LA AMAZONIA PERUANA
}

\author{
Walter Mertens
}

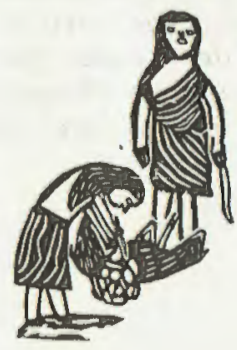

Il y a, pourt'la uteur, des attitudes de base, si l'on veut envisager un type quelconque de développement de l'Amazonie.

Il faut tout d'abord étudier la réalité amazonienne; puis agir d'une facon conforme aux conclusions établies par les études effectues dans ce domaine. En même temps, il indispensable de respecter l'identité culturelle des habitants de l'Amazonie, forgée à travers les siècles.

On expose dans, cet article les différentes politeques de développement practiqueés dans cette région du Pérou. Certaines ont essayé de faire de l'Amazonie une parte intégrale de la nation Pérouvienne. D'autres, au contraire, la considèrent comme un territoire de provicions $q u$ on pout explorer et exploiter en temps voulu.
The author feels that, in order to plan any kind of development in the Amazon, certain basic attitudes should be assumed: study of the Amazonian situation and action coherent with its conclusions, and respect for a cultural identity that has been forged throug. hout the centuries.

This article is an exposition of diverse policies that have been proposed and put into practice with respect to development in this region of Peru. Throughout the article we can see how some of these policies have tried and do try to consider the Amazon integrally as a part of the Peruvian nation, and how others have considered the region as the bread-basket of the country and a territory that can be invaded and exploited at any given time.

* Walter Mertens escribió este artículo durante el Gobierno de Fernando Belaúnde Terry. Amazonía Peruana considera que es un valioso aporte, aún vigente para la comprensión de lo que la aplicación de diferentes políticas han significado para la región, dando como resultado lo que hoy es nuestra Amazonía. 
In diesem Artikel werden die unterschiedlichen Politiken und Haltungen im Hinblick auf die Entwicklung des peruanischen A mazonas-Raumes dargestellt, der hier als Teil der Nation gesehen wird und nicht als das gleichgültige und verlassene Geisterreich, das vielfach auch als "Speicher" des Landes gilt, auf den man im gegebenen Moment zurückgreift, um ihn entsprechend $z u$ plïndern.
Für die Entwicklungsplanung in die ser Region ist es unerlässlich, die Realität zu studieren und den Ergebnissen entsprechend $z u$ handeln, wobei die wirtschaftliche und soziale Identität $z u$ respekticren ist, die die Bevölkerung in Jahrhunderten herausgebildet hut. Freilich sollte man dabei das Wie nicht aus den Augen verlieren angesichts der überaus vielschichtigen Problematik.

De los países amazónicos, el Perú es el que tiene la mayor proporción de su territorio en zona amazónica, propiamente dicha. Aproximadamente $60 \mathrm{o} / \mathrm{o}$ del área territorial del Perú es amazónica. Sin embargo, Brasil con más o menos 55 o/o del total de área amazónica en su territorio, alberga la mayor parte del Amazonas.

Aunque el Amazonas Peruano es tan sólo una séptima parte del Amazonas Brasilero, es sin embargo un área impresionantemente grande, en la cual fácilmente cabrían las superficies combinadas de Alemania Occidental, Austria, Italia, los Países Bajos, Suiza e Israel (1).

En comparación con Brasil, la Amazonía recibe en el Perú mucho menos atención de la comunidad nacional. El Perú realiza menos investigación sobre la Amazonía que el Brasil. En la mentalidad popular, sin embargo, la Amazonía es el objeto de discusiones que muchas veces toman cuerpo en sueños míticos, mezclados con relám. pagos de una visión interna más realista. La población es siempre un ingrediente importante en cualquier debate sobre la Amazonía en el Perú. Uno podría incluso afirmar que la población es el mayor componente de cualquier discusión peruana, donde el tema de las potencialidades demográficas de la Amazonía es materia de discusión. Diferentes perspectivas asomarán en estas discusiones, algunas simples, otras sofisticadas. Algunas han sido impresas, otras sólo tienen dimensión oral. En este trabajo se hace un esfuerzo para construir una tipología de ideologías de la población amazónica del Perú.

Se distinguen dos tipos principales y mutuamente excluyentes: Por un lado existen los "expansionistas", cuyo tenor principal es que la Amazonía necesita una población grande y de rápido crecimiento. Por otra parte existen los "prudencialistas", quienes tienen una visión más cautelosa de capacidad de asentamiento de población en la Amazonía. Dentro de ambos tipos, podemos distinguir varios sub-grupos que reflejan diferencias en orientación, énfasis y estilo. Estos sub-grupos no son necesariamente excluyentes, $y$ en el trabajo de un mismo autor podemos encontrar orientaciones diversas, siempre dentro de uno de los dos grupos principales. Estos sub -grupos son representados esquemáticamente en el siguiente cuadro: 


\title{
Síntesis de Ideologías Amazónicas de Población
}

\author{
a. Expansionistas: \\ 1. Expansionistas Simples \\ 2. Solucionadores de Problemas \\ 3. Geopoliticos \\ 4. Supra-nacionalistas.
}

b. Prudencialistas:

1. Indigenistas

2. Ecologistas

3. Pesimistas de la Colonización.

Entre los expansionistas se distinguen 4 grupos: los expansionistas simples, quienes sienten que la Amazonia puede y debería tener una población mayor para poder desarrollarla; los solucionadores de problemas, quienes ven el Amazonas como un instrumento principal para resolver el problema de la población,asi como otros problemas del Perú. Tenemos luego los geopolíticos, quienes están a favor de una gran población en la Amazonía como respuesta a la presión de los países fronterizos; y finalmente los supranacionalistas, quienes piensan que la Amazonía peruana debería ser desarrollada dentro de una estructura de cooperación inter-regional amazónica, contribuyendo así a la solución de la población, no sólo en Perú sino en otras regiones con este mismo problema.

Los prudencialistas se dividen en 3 grupos: los Indigenistas, quienes se oponen a la colonización de la Amazonía por los problemas que esto ocasionaría a la población indígena original. Luego están los ecologistas, que se basan en los recursos limitados pa. ra la agricultura intensiva, asi como en el deterioro del suelo y medio ambiente causado por la migración. Y finalmente los pesimistas de la colonización, quienes señalan la experiencia desdichada de la colonización planificada como una razón para tener prudencia.

Las diferentes orientaciones presentadas en el cuadro, son elementos en el presente debate sobre población Amazónica en el Perú. Históricamente el primer grupo, los expansionistas, ha sido mucho más prominente. Es tan sólo recientemente que el segundo grupo ha hecho sentir su presencia en al debate.

Este trabajo no está dirigido a la discusión de las ideologías de la población ama. zónica del pasado, lo cual podría ser tema de un estudio interesante; el énfasis está puesto en el análisis del pensamiento peruano actual sobre la población y la Amazonía Sin embargo, ocasionalmente nos referimos a declaraciones menos recientes. La discusión se basa, principalmente, en el análisis de varios trabajos y libros publicados en años recientes sobre la Amazonía peruana, y en una serie de entrevistas con estudiosos y representantes del gobierno interesados en la Amazonía. Entre las publicaciones recientes, prestamos una atención especial a los trabajos presentados en un Fórum sobre la Amazonía organizado en octubre de 1978, por el Colegio de Arquitectos del Perú (2).

El trabajo ha sido dividido en varias partes, de acuerdo a la categorización previa. mente discutida. Antes de iniciar una discusión detallada de las diferentes ideologías, dedicaremos unos párrafos introductorios a los problemas de definición y a la discusión de algunas características de la Amazonia peruana. 


\section{UN PROBLEMA DE DEFINICION}

En esta sección se discute el problema de definición y se presenta alguna informaciỏn socioeconómica y demográfica de la Amazonía peruana.

¿A qué nos referimos con Amazonía Peruana? La Amazonía peruana incluye el territorio íntegro de una de las tres áreas ecológicas en las cuales el Perú se divide tra. dicionalmente: la estrecha Costa del Pacífico, la sierra o Montaña, y en el Oriente, la selva La Amazonía peruana entonces, coincide con lo que se llama la selva en el Perú. Por lo tanto, en este trabajo los dos términos, Amazonía peruana y selva, se utilizarán indistintamente ya que se refieren a la misma área exáctamente. Esta área contiene la totalidad de los departamentos de Amazonas, San Martín, Loreto, Madre de Dios, así como parte de otros departamentos. Aquí radica un poco más del $100 / 0$ de la población del Perú.

En el Perú se hace siempre una distinción entre Ceja de Selva y la selva propiamente dicha. El término Ceja de Selva se refiere a las laderas orientales de los Andes, así como a los valles intermedios; llamada también Selva Alta, su altitud varía entre 2,000 y $1,000 \mathrm{mts}$. a $400 \mathrm{mts}$. (3) y en contraste con las áridas laderas occidentales de los Andes, está cubierta de vegetación tropical, y cruzada por una gruesa red de ríos. Esta distinción refleja importantes referencias ecológicas. La Ceja de Selva permite un grado de explotación agrícola imposible en la selva misma, donde la economía siempre se ha basado en modos de producción extractivos. Esta distinción tiene también una importancia demográfica en tres aspectos: primero, la densidad demográfica de la Ceja de Selva es más alta que la de selva , la Ceja de Selva tiene casi el doble de población que las áreas bajas. La migración de campesinos de la sierra a la Amazonía peruana, se dirige especialmente a la Ceja de Selva. Estas partes se prestan más al cultivo de productos co. mo el azúcar, té, cacao, coco, y tabaco. En segundo lugar, las diferencias ecológicas han sido importantes para el destino demográfico de los grupos étnicos nativos de la Amazonía. Demográficamente los habitantes nativos de la Ceja de Selva se han podido defender mejor, ya que el medio ambiente les ha permitido llevar una vida más estable, de una manera demográficamente más concentrada. La ecología de la selva, por el contrario, ha favorecido la dispersión en pequeños grupos, y aunque esa dispersión muchas veces ha servido de protección contra la intrución cultural; también ha significado que los nativos puedan ser fácilmente controlados y exterminados por intrusos (4). En tercer lugar, en las discusiones sobre asentamientos de población potenciales en la selva, con frecuencia se enfatiza que la Amazonía peruana es diferente de la Amazonía brasileña en su constitución ecológica, y que las decepciones en la experiencia de colonización en la Amazonía brasileña no se pueden extender a la Amazonía peruana, debido a las mayores posibilidades de asentamiento de población en la Ceja de Selva.

Dentro de la Selva, los antropólogos hacen aún otra distinción entre la varzea y la tierra firme. La varsea es una llanura inundable que aumenta su importancia a medida que uno se acerca al delta del Amazonas. La tierra aquí es rejuvenecida anualmente con el fértil sedimento andino, y por lo tanto permite una explotación agrícola sustancial. La tierra firme consiste en las áreas entre los ríos, las cuales no están sujetas a inundaciones. Aquí los recursos son escasos pero más estables que en la varzea, en donde la abundancia depende de las variantes estacionales del río. Los diferentes sistemas ecológicos de las dos áreas han resultado, en diferentes sistemas culturales, una mayor densidad de población en las llanuras inundables (5). Esta diferencia en densidad demográfica se ha mantenido en tiempos actuales, debido a la importancia de la red fluvial en la comercialización y el transporte de la Selva. 


\section{EL PUNTO DE VISTA EXPANSIONISTA.}

\section{Expansionistas simples.}

La perspectiva expansionista, de que la selva está ahí en espera de ser poblada y desarrollada, ha sido probablemente la más dominante, si no la única en el pasado. Con frecuencia toma tonos nacionalistas. Aunque hoy pierde importancia, aún se pueden encontrar suficientes argumentos a favor como para que sea prematuro relegar esta perspectiva a la antigüedad. La perspectiva expansionista simple, aún se reflejaba en las declaraciones de algunos trabajos del Fórum sobre la Amazonía. Un trabajo afirma de manera aprobatoria que: "Las condiciones naturales de la Amazonía, podrían conllevar a un aumento tremendo de población" y cita, como apoyo a esta teoría, el alto ritmo de crecimiento en la Amazonía brasileña (6). Otro trabajo del mismo Fórum afirma que: "... no hay mayor beneficio para el país, que un peruano trabaje y forje su tierra en un pedazo de la selva" (7).

La opinión de que la Amazonía peruana está vacía y debe ser poblada, casi siem. pre va acompañada de la convicción de que este vacío demográfico representa un serio problema de desequilibrio poblacional en el Perú. El análisis de los problemas sociales, económicos y políticos del Perú en términos de desequilibrio regional, especialmente desde el famoso ensayo de José Carlos Mariátegui sobre regionalismo y centralismo en el Perú (8), es una característica frecuente de los análisis sociológicos de la sociedad peruana. El desequilibrio demográfico es tan sólo un aspecto de esto, y no es mencionado siempre en este contexto.

La visión expansionista simple, en especial en el siglo XIX, dió lugar a medidas políticas, favoreciendo la colonización de la Amazonía, particularmente por inmigrantes europeos. Algunos de estos incentivos han sido la exoneración temporal de impuestos, tierra gratis, ciudadanía peruana, pagos especiales por el transporte y el período inicial de asentamiento, e incluso pagos incentivadores a los intermediarios que trajeran inmigrantes al Perú (9).

La preferencia por inmigrantes europeos, se justificó con frecuencia con la teoría de que sólo las personas provenientes de culturas "superiores" podrían ser capaces de explotar los recursos de la selva (10).

\section{Los solucionadores de problemas}

Fue un proceso natural el hecho que el punto de vista expansionista simple, se traduzca a una visión del Amazonas como una solución para reubicar el excedente de población resultante del alto índice de crecimiento natural del Perú. De esta manera, existe un cambio en el énfasis. Mientras que en el pasado la Amazonía representaba simplemente un vasto potencial para la expansión demográfica, sin ninguna referencia a los problemas de población; ahora se aprecia el hecho de que haya un problema de crecimiento de población en el Perú, y que éste puede ser solucionado por medio de inmensos traslados de población de las áreas más pobladas hacia el Amazonas peruano. En la prensa popular no es poco común el encontrar afirmaciones como que la solución a cualquier problema mayor del país depende del desarrollo de la selva, lo cual implica un crecimiento simultáneo de su población.

El concepto de la colonización de la selva, como una válvula de escape para aliviar problemas agrícolas y presiones poblacionales, no es exclusivo del Perú. Esta idea se puede encontrar en otros países Latinoamericanos, entre los cuales Brasil es el más notorio (11). 
En otras partes del mundo, a veces se han justificado políticas similares como en el programa de transmigración de Indonesia (1.2).

Tres problemas específicos e interconectados se citan con frecuencia como apoyo a la opinión que poblar la Amazonía resolverá el problema de población del Perú.

Primero, la colonización de la selva resolverá el problema de los campesinos sin tierras de la sierra; problema causado por el rápido crecimiento demográfico en un contexto de estructuras agrarias anticuadas. En segundo lugar, la colonización de la selva reducirá la migración hacia las ciudades de la costa. En tercer lugar, la transferen. cia de poblaciones rurales marginales, especialmente de las ciudades de la costa, podrían reducir la presión del crecimiento urbano de las áreas costeras del Perú.

Las medidas adoptadas en el pasado para la Reforma Agraria, frecuentemente han incluido planes de colonización de la selva, muchas veces como sustituto de un verdadero cambio estructural en la tenencia agrícola (13). Por ejemplo, el ahora desaparecido Instituto para la Reforma Agraria y Colonización (IRAC), no fue aprobado por el Congreso, quien solo proveyó fondos para un limitado programa de colonización de la selva, y algunas obras públicas en áreas rurales que habían experimentado una seria agitación campesina (14).

Estas ideas se encuentran con frecuencia en las palabras y programas del ex-presidente Fernando Belaúnde Terry. En el discurso inaugural al Fórum, Belaúnde muestra que no ha perdido su antigua fe en el desarrollo de la selva como una solución al problema demográfico del Perú. En este discurso, repite su antiguo énfasis en la necesidad de intensificar la colonización de la Amazonía peruana, subrayando su rol en la solución de problemas resultantes del rápido crecimiento de la población en la sierra y el crecimiento de las "barriadas" en las ciudades costeras del Perú. Belaúnde a menudo justifica su política de construcción de carreteras en la selva -en especial la "Carretera Marginal" (15), uniendo los diferentes valles tropicales de la Ceja de Selva-como medidas para aliviar la presión demográfica, para asentar a los campesinos sin tierra de la sierra, y para contrarestar la migración a la costa (16).

Aunque Belaúnde no estaba en contra de la Reforma Agraria como principio "sus escritos sugieren que prefería construir carreteras de penetración a las nuevas tierras como una manera de mejorar el radio hombre-tierra sin perjudicar a nadie". (17).

Tampoco significa que Belaúnde se oponía a otras políticas de población (18), como lo nuestra el hecho de reconocer las serias implicancias del rápido crecimiento demográfico, y que su gobierno aprobara la inclusión de la planificación familiar dentro de los programas de salud materno-infantil. Su gobierno incluso firmó un acuerdo con la organización Panamericana de la Salud en 1968 para este programa, el cual nunca se pudo llevar a cabo por el cambio de gobierno. Sin embargo, el primer gobierno de Belaúnde no tuvo una verdadera política de población, como observa Juan Wielt en un excelente estudio sobre la situación demográfica del Perú (19).

En cuanto a la reforma agraria, no hay duda de que su preferencia yace en la redistribución de la población hacia la selva. Que Belaúnde no ha cambiạdo su punto de vista a este respecto, está claro en el artículo que publicó en un importante diario limeño (20); en el cual enfatizaba las ventajas climatológicas y topográficas de la Selva. Alta para asentamientos humanos, y las "condiciones óptimas de la Selva Alta para atraer el exceso de población que hoy congestiona nuestras ciudades. Es por esto que he dicho, y no me cansaré de decir: creer en la selva no es una obsesión, es una solución".

El reciente torbellino de atención dada por Belaúnde al desarrollo de la selva en su nuevo gobierno, nos confirma que la expansión demográfica y económica de la selva 
sigue siendo un item importante en su agenda. El reinicio de los trabajos de construcción de la Carretera Marginal ha sido declarado una obra de necesidad y utilidad pública (21). En un discurso reciente, Belaúnde prometió que el gobiemo desarrollaría un "eje colonizador" en la Selva Central (22). Un editorial reciente de el diario "El Comercio" apoya esta política y detalla que "la gran reserva de América Latina está situada en el húmedo trópico", y que fácilmente se puede acomodar a un millón de habitantes en el Valle del Huallaga (23).

Tanto los expansionistas simples como los solucionadores de problemas, generalmente tienen una tremenda confianza en la disponibilidad de los inmensos recursos naturales de la Amazonía, y la creencia en que la tecnología y la ciencia pueden solucionar cualquier problema que se suscite en el camino hacia la expansión demográfica de la Amazonía.

Héctor Vargas Haya, un aprista director del Comité de Asuntos Amazónicos de la Asamblea Constitucional, escribe que "El Amazonas es una región privilegiada donde todo abunda. (24). La región Amazónica es tan fértil como la tierra más fértil y nada es imposible en vista del desarrollo tecnológico" (25).

La Amazonía es un "gigante durmiente", el cual "no puede permanecer en su actual estado primitivo con sólo unos cuantos centros poblados que se pierden en la inmensidad de la selva", (26). El mismo autor está superficialmente familiarizado con la más reciente crítica de los prudencialistas, basada en estudios ecológicos, pero los descarta por "artificiales y sin valor científico, productos de la irresponsabilidad, la improvisación y el empirismo", (27).

Con frecuencia, la falta de una política adecuada es considerada la razón princi pal del por qué la selva no tiene un mayor desarrollo y población. En un documènto preparado por el Ministerio de Vivienda encontramos la siguiente declaración:

"Es de notar que la inhabilidad de la selva para atraer inmigrantes, reflejada en el pequeño volumen de migración a la selva, constituye un serio problema de desequilibrio de la sociedad peruana. En efecto, en vista de la existencia del gran territorio amazónico, su escasa población, y la disponibilidad de recursos naturales, esta área debería ser el centro de recepción, por excelencia, del exceso de población de otras zonas del país. El que esto no haya sucedido asi, es culpa de los lideres políticos quienes han hecho muy poco por el desarrollo de estas áreas, condenándolas a una situación de abandono" (28).

Héctor Vargas Haya considera "la falta de un verdadero estímulo financiero y tributario" (29), como el talón de Aquiles del progreso amazónico. El hecho que muchos asentamientos de población nazcan y mueran de manera anárquica, se explica por "la ausencia de una organización política de centros urbanos con objetivos definidos" (30).

Con frecuencia se piensa que el promover la selva como una solución a los problemas del Perú, es una característica de los partidos políticos de derecha. Sin embargo, una cuidadosa observación del panorama político del Perú, nos muestra que este punto de vista recorre el espectro político entero. A Genaro Ledesma, líder de una facción de los izquierdistas, se le planteó durante una entrevista en televisión, la posibilidad que su propuesta política de suspender el pago de la deuda externa, pusiera en peligro la obtención de créditos extranjeros necesarios para la compra de productos alimenticios. A esto contestó que él reforzaría el desarrollo agricultural de la selva, $\mathrm{y}$ así en corto tiempo el Perú podría ser autosuficiente en cuanto a comida. En otra ocasión declaró que, con la migración de las áreas urbanas hacia la Ceja de Selva de miles de obreros desempleados, Lima y otras grandes ciudades se verían inundadas por la abundante cosecha de fruta (31). 
En entrevistas a varios políticos peruanos y conocedores (32), en las cuales se les preguntaba si el crecimiento demográfico del Perú debía ser estimulado o limitado; Ledesma se declarará a favor del estímulo del crecimiento de población, ya que el Perú tiene un inmenso, aunque escasamente habitado territorio. Sin embargo, para mantener a esta población creciente - contrario a declaraciones anteriores- ve menos posibilidades en la agricultura, poniendo énfasis, en cambio, en el desarrollo de recursos naturales.

\section{Geopolíticos}

La experiencia histórica ha hecho que el Perú se preocupe mucho por sus áreas fronterizas. Hace cien años, en la guerra del Pacífico, Chile le quitó parte'de su territorio. Durante la guerra con el Ecuador, se anexó parte de la Amazonía ecuatoriana, hecho que aún afecta las relaciones entre ambos países.

Aunque nunca ha existido una guerra entre Perú y Brasil, la historia de las relaciones peruano-brasileras se ha caracterizado por una continua expansión política y demográfica hacia el oeste (33). Es más, la cesión a Brasil de parte de la selva denunciada por Perú en 1908, indujo al Presidente Leguía a promover la colonización amazónica con inmigrantes extranjeros.

La preocupación geopolítica con las áreas fronterizas se ha convertido así en una característica predominante de las relaciones del Perú con varios de sus vecinos. Esta preocupación se ha traducido en un deseo de aumentar la densidad de población de las áreas fronterizas, lo cual se ha convertido ahora en política oficial.

El Centro de Altos Estudios Militares, centró su atención en este tema en 1958, con la publicación de un plan de colonización a gran escala usando personal civil y militar (34). Entre las actividades para el desarrollo regional, un item importante es el apoyo político a las áreas fronterizas. El documento preparado por el Instituto de Planificación Nacional con ocasión de la Primera Conferencia Mundial de Población de Budapest, establece que los patrones de distribución de población son importantes para la seguridad nacional y el desarrollo, y por lo tanto las medidas para poblar áreas fronterizas, son parte de la política para una mejor distribución de la población en el territorio nacional (35).

El Plan Nacional 1977-1978, incluye la creación de una infraestructura y servicios a las áreas fronterizas y "el establecimiento de un sistema racional de centros de población, en función de las actividades que se desarrolle en cada uno de ellos" (36).

E1 Plan Nacional de Desarrollo 1979-1980 (37), como el Plan anterior, tiene toda una sección de recomendaciones para el desarrollo de zonas de frontera económica en el capítulo sobre organización territorial. Estas recomendaciones se refieren a diversos proyectos de desarrollo de infraestructura; construcción de carreteras y explotación de recursos naturales, que deben tener un impacto sobre los asentamientos humanos. Otras se refieren directamente a los proyectos de asentamientos humanos; aunque en el Plan de Desarrollo 1978-1979., el énfasis se pone más en los estudios que en la acción directa. El Ministerio de Educación también empieza a participar. Durante un Seminario (38) organizado por el Ministerio en 1979, se presentó un plan para dar la cobertura educativa máxima a la población de las áreas fronterizas.

En el Fórum había una preocupación general respecto a la geopolítica, siempre dirigida a los brasileños (39). Esta preocupación se hizo sentir especialmente a través de numerosas preguntas, algunas bastante delicadas, dirigidas a la delegación brasileña (40) en el Fórum. Se puso en duda la buena intención de la construcción de la red brasileña de carreteras amazónicas. 
"¿Por qué los brasileños quieren establecer tanta gente en estas regiones?" "No es la historia del Brasil una expansión contínua hacia el Oeste?" "No fue demasiado favorable al Brasil el último tratado amazónico de amistad?" Para algunos, esta preocupación se hizo sentir también en los pedidos de acelerar el establecimiento de poblaciones en las zonas fronterizas del Perú, especialmente en el límite con Brasil. Uno de los trabajos presentados al.Fórum (41), criticaba específicamente el Tratado Amazónico firmado por los países amazónicos por poner en peligro, indirectamente, la soberanía del Perú. Debido al mayor nivel de desarrollo del Brasil, el artículo que establece la libertad de navegación en el sistema del Río Amazonas favorecería la penetración bra. sileña en el Perú. Entre las varias recomendaciones que el Fórum presentó al gobier. no, hay una que expresa muy explícitamente estas preocupaciones geopolíticas:

"Es una necesidad prioritaria proveer una infraestructura de carreteras con obje. tivos geopolíticos para el conjunto de la Amazonía, y estimular y acelerar el desarrollo de nuestras provincias fronterizas para detener la penetración de los países vecinos" (42).

Preocupaciones semejantes se expresaron en el Primer Seminario Sobre Geopolítica y Estrategia en setiembre y diciembre de 1978, organizado por el recientemente conformado Instituto Peruano de Estudios Geopolíticos y Estratégicos. En este Seminario, (43) Hermann Buse, en una charla sobre zonas de tensión fronteriza en el Perú, señala el avance brasileño "antes espontåneo, hoy organizado" hacia Paraguay, Bolivia y Perú; basado en la diplomacia del principio de "util possidetis de facto" y en el concepto de fronteras móviles.

El mismo Centro publica una nueva revista dedicada a los estudios geopolíticos y estratégicos. A la fecha, han aparecido cuatro números que traen regularmente artículos sobre el expanșionismo brasileño y la importancia geopolítica, crucial para el Perú, de desarrollar su territorio amazónico. Entre éstos, un artículo de Edgardo Mer. cado Jarrín, director del Instituto, describe la innegable expansión del Brasil hacia el Océano Pacífico y considera al Pacto Andino como un medio importante para controlar esta expansión. Expresa también cierto recelo acerca de si el Tratado Amazónico recientemente firmado con Brasil se convertirá en otro instrumento de expansionismo (44).

Otro artículo en la misma revista subraya que el mandato del Tratado Amazónico de "promover el desarrollo de las respectivas zonas fronterizas sobre la base de la colaboración", debe ser un aliciente para que el Perú persiga un desarrollo más activo de sus áreas fronterizas frente a la política amazónica brasileña" (45).

En un artículo más reciente, dedicado exclusivamente a un análisis del Tratado Amazónico, Mercado Jarrín adopta una óptica más positiva respecto al tratado. Lo ve, en parte, como resultado de la necesidad de los países andinos de hacer frente común ante el Brasil. Señalando que la Amazonía brasileña no es más un espacio vacio y que, debido a su rápido crecimiento poblacional, ejerce presión natural sobre las áreas más débiles -económica y demográficamente-de los otros países, dice:

"El pacto amazónico, debe convertirse en estímulo para la movilización efectiva de parte de cada miembro firmante, para promover medidas dinámicas y efecti. vas para colonizar y poblar sus respectivas áreas amazónicas, en previsión de las inevitables carreteras comunicantes que se establecerản con el Brasil" (46).

Advirtiendo especificamente al Perú contra la avalancha demográfica brasileña que ha puesto mucha presión sobre el departamento Madre de Dios, a firma:

"Es fundamental que el Perú desarrolle una geoestrategia de comunicaciones que rectifique la falta de ocupación demográfica de la Amazonía, que reoriente el pro. 
ceso migratorio, que ponga énfasis especialmente en los medios no convencionales de transporte, colonice y nacionalice sus áreas fronterizas..." (47).

Inclusive se pueden encontrar afirmaciones como que la migración interna, predominantemente dirigida hacia la costa y hạcia Lima, debilita más las áreas fronterizas del Perú. Roel, un economista con influencia en algunos medios peruanos, escribe:

"La monstruosa concentración demográfica en Lima ha ocasionado la deserción relativa del resto del país, con el efecto doblemente negativo de que las áreas demográficamente desiertas son una invitación a la penetración foránea y que, por añadidura, estas áreas se hacen difíciles de defender sin apoyo externo" (48).

Hasta se propone que el rápido crecimiento urbano de Lima debe y puede ser neutralizado por grandes transferencias de población a las áreas fronterizas, en lugar de una política que ponga atención a las variables de fertilidad. Emilio Castañón Pasquel, en el tercer número del periódico dedicado a la geopolítica antes indicado, habla sobre:

"... el hecho absurdo de que en el Perú más de 4 millones de personas viven en el valle de Lima en una situación que se podría considerar como de sobre población, mientras que hay extensas áreas subpobladas a lo largo de la frontera con Brasil. Debido a esto, es ambiguo - por decir lo menos - hablar de la necesidad de controlar la explosión demográfica en el valle de Lima con un programa de paternidad responsable; mientras que los valles del interior del país más allá de Lima están vacíos, sin visualizar un programa de colonización. En términos generales, es necesario resaltar que es más fácil reducir la población limeña en dos millones, en el lapso de diez años, mediante la colonización del interior que mediante un programa de control de la natalidad" (49).

Edgardo Mercado Jarrín, al discutir la necesidad de desarrollar la selva central del Perú como zona geoestratégica de primera importancia y de óptimo desarrollo económico, dice: "La Selva Central se presenta a la nación peruana como un vacío espacial vasto, extenso y transversal, naturalmente dispuesto para absorber buena parte de las corrientes migratorias hacia Lima y su expansión natural" (50).

Hay poca o ninguna información disponible, acerca de si el gobierno ha tenido éxito en aumentar la población de estas áreas fronterizas. Un artículo reciente en un semanario serio peruano, sugiere que el gobierno no logra siquiera retener a la población original de estas zonas (51). Otros observadores me indicaron que dichos asentamientos fronterizos, pueden tener un efecto exactamente contrario: promover la penetración económica y cultural brasileña en las áreas fronterizas amazónicas del Perú. Los colo. noş están siendo absorbidos por el sistema económico brasileño y, en algunos lugares, la moneda empleada en transacciones locales no es el sol sino el cruzeiro. El idioma gradualmente se inclina al portugués, a medida que las familias colonas envían a sus hijos a escuelas brasileñas.

\section{La visión supranacionalista}

La colonización de la Amazonía peruana usualmente se concibe de manera muy nacionalista, El énfasis se pone en el desarrollo nacional del Perú y en los intereses geo. políticos peruanos. En un momento en que en varias partes de Sudamérica la preocupación nacionalista estrecha parece estar cobrando fuerza, es refrescante ver que en el debate amazónico algunas personas adoptan una visión supranacionalista. Aunque como se ha visto en el libro de Héctor Vargas Haya (52), existen muchas debilidades, tiene la ventaja que en la discusión de la Amazonía peruana, él asume la óptica de que sólo a través de la coordinación e integración de las políticas amazónicas se logrará el desa- 
rrollo de la Amazonía. Critica con razón las políticas frecuentemente contradictorias seguidas por los diversos países amazónicos de América Latina, y promueve la creación de una Organización Regional Amazónica, semejante al Pacto Andino. El autor está evidentemente influido por una de las consignas apristas; la necesidad de transformar América Latina en los Estados Indoamericanos Unidos. Desgraciadamente, esta óptica supranacionalista se ve empañada por las mismas características ingenuas presentes en toda la obra. La integración deberá acompañarse de una red amazónica de ferrocarriles; un canal sudamericano que una las cuencas del Amazonas, El Orinoco y el Plata (53); y la creación de un gran lago amazónico como sugiere el proyecto preparado por el Instituto Hudson.

Además de una simple visión expansionista, en un marco supranacionalista, algunos autores peruanos consideran a la Amazonía como la solución a los problemas cau. sados por el rápido crecimiento poblacional no sólo del Perú, sino de América Latina. En un documento (54) de Hernando Quintana Arriola, que describe bien algunas facetas del problema poblacional latinoamericano y menciona que América Latina "tiene el dudoso honor de ser la campeona de la explosión demográfica", se puede leer también:

"... dónde entonces podemos hallar el espacio amplio y la potencialidad en recur-

sos naturales para sostener la vida de tantos millones de personas. Desde mi punto de vista, sólo hay una respuesta. El gran trópico húmedo de América Latina".

El autor continúa en una vena casi mítica: la verdadera América Latina se desarrollará en las llanuras tropicales y al hacerlo, América Latina diseñará finalmente su propia tecnologia, diferente de los modelos norteamericanos y europeos.

Tomando un punto de vista supranacional, Héctor Vargas Haya concibe a la Ama. zonía como solución al rápido crecimiento pobilacional mundial (55). Millones pueden afincarse en la Amazonía y, a la vez, el potencial agrícola amazónico puede desarrollarse para alimentar a las enormes poblaciones que resulten del rápido crecimiento demográfico mundial. Aún cuando se acepte la planificación familiar, la población mundial aumentará y por lo tanto será conveniente que esta población creciente tenga acceso a los recursos amazónicos nacionales.

El autor resume bien su punto de vista en el siguiente părrafo:

"La población excedente de otras latitudes como China o India, incluyendo a Europa (sic), nos ofrece un acertijo a ser resuelto por estadistas y científicos. La riqueza amazónica está ahí: extensa, majestuosa, esperando que el hombre y la tecnología la transformen en la solución al complejo problema de espacio y alimento para el mundo" (56).

Sin embargo, el libro no trata de todos los problemas. prácticos de cómo ubicar a estos millones en la Amazonía, cómo crear empleo para ellos, y cómo integrarlos al medio cultural latinoamericano.

\section{LA VISION PRUDENCIALISTA}

La ideología prudencialista es de origen más reciente, pero es posible encontrar posiciones prudencialistas en el pasado. El fracaso de muchos intentos de colonizar la selva durante el siglo XIX y el presente, ha sembrado ocasionalmente semillas de duda (57). Las recurrentes experiencias frustrantes de explotación de recursos particulares. como el caucho, periódicamen te ha apaga do el optimismo expansionista. 
El mero volumen y aparente inexorabilidad de la corriente migratoria hacia la costa también ha sido un factor que, crecientemente durante el siglo XX, ha llevado a algunos peruanos a pensar dos veces las posibilidades de colonizar la Amazonía. Esta actitud se refleja aún en el pensamiento gubernamental. Según un informe de las Naciones Unidas sobre las políticas de población en el Perú, "el gobierno considera que la zona costeña seguirá como generadora esencial del crecimiento económico y como área de mayor atracción de migrantes" (58). Sin embargo, es justo decir que sólo en tiempos muy recientes la visión prudencialista ha cobrado una influencia real en el campo de las ideologías de desarrollo y población amazónica. Este punto de vista está aún limitado a grupos de expertos y cientificos que han estudiado en detalle la Amazonia. Su peso en las decisiones gubernamentales sigue siendo débil, pero está creciendo.

Se pueden distinguir tres corrientes prudencialistas. La primera tiene sus orígenes en una preocupación por los grupos de población indigena de la Amazonía; la segunda deviene de una mejor comprensión de la ecología amazónica; y la tercera de estudios recientes y detallados de los esfuerzos de colonización. En aras de la brevedad llamaremos a los tres grupos, respectivamente, indigenistas, ecologistas y pesimistas de la colonización. Las tres corrientes están muy interrelacionadas. Indigenistas y ecologistas, a menudo resaltarán el admirable equilibrio ecológico alcanzado en el pasado por los grupos indígenas amazónicos. Los pesimistas de la colonización a menudo toman esta actitud debido a los efectos negativos de la colonización sobre los grupos nativos y sobre el medio ambiente de la Amazonia.

Debe resaltarse desde un comienzo, que la visión prudencialista no necesariamente implica la oposición al desarrollo de la Amazonía, la explotación de sus recursos naturales y de otro tipo, ni la promoción de asentamientos humanos. Aunque ocasional. mente se oyen posturas extremas de este tipo entre algunas corrientes de pensamiento indigenista, la corriente prudencialista mayoritaria en el Perú ciertamente no está en contra del desarrollo amazónico. Sin embargo, normalmente subrayan dos elementos importantes. Primero, que dichas políticas deben basarse en la investigación exhaustiva, y segundo, que las posibilidades de colonización y desarrollo amazónico son mucho más limitadas de lo que se imaginaba en el pasado.

\section{Indigenistas}

Llamamos ideologías indigenistas de población amazónica a aquellas que subrayan que toda política de desarrollo y colonización amazónica debe prestar atención prioritaria a los nativos de la Amazonia. Dentro de este grupo distinguimos tres énfasis diferentes: utópicos, nostálgicos del Tahuantinsuyo, y defensores de los derechos humanos. La posición utópica se basa en la observación de que los nativos de la Amazonía, a través de su cultura y sociedad, han desarrollado un notable equilibrio ecológico con su medio ambiente. Este equilibrio debe perturbarse lo menos posible. En su forma más extrema, rara vez expresada por escrito, la posición utópica sostiene que la Amazonía debe dejársele exclusivamente a los grupos nativos y que toda política de desa. rrollo amazónico es errónea. Esta posición es denominada a veces despectivamente como de "museo cultural" por sus críticos, ya que sus partidarios supuestamente qujsieran mantener a los grupos étnicos originales de la Amazonía como en una vitrina de un museo de antropología cultural (59).

Los nostálgicos del Tahuantinsuyo parten de una premisa algo diferente. Ellos enfatizan que el Tahuantinsuyo -el antiguo imperio incaico - al revés de lo que pien. san muchos, tuvo contacto y relación frecuente e intensa con los pueblos amazónícos. 
Dicho contacto y relación fueron resultado de la genialidad administrativa de los Incas, y es sobre esta experiencia (que incluía la percepción de la precaria ecología del medio tropical) que deben fundarse las políticas de desarrollo amazónico. (60). Finalmente. hay quienes aceptan que es deseable el desarrollo amazónico, pero subrayan que los nativos frecuentemente han sido víctimas de las políticas de desarrollo y colonización, y que en el futuro la política debe diseñarse integramente para proteger sus derechos. Este énfasis en los derechos de las comunidades nativas de la Amazonía se comparte con las otras posiciones, especialmente la utópica (61).

El énfasis en las condiciones de los grupos étnicos originales en el debate sobre colonización amazónica, pasó a primer plano especialmente a fines de los años 60 , con el surgimiento de lo que Manuel Marzal llama el "indigenismo crítico" (62). Este nuevo tipo de indigenismo reconoce elementos positivos en los tipos anteriores de indigenismo. pero también critica las visiones previas, por enfatizar demasiado la integración de los indígenas a la sociedad nacional peruana. Los indigenistas actuales sienten que las políticas diseñadas en el marco anterior, pueden finalmente favorecer la desaparición de las culturas nativas, produciendo asi un "etnocidio cultural"; como se llama al proceso en las discusiones acaloradas. Dos antropólogos culturales peruanos, Alberto Chirif y Stefano Varese, han sido especialmente elocuentes para traer al debate esta perspectiva. Alberto Chirif escribió un interesante documento sobre la historia de la ocupación territorial de la Amazonía desde la llegada de los españoles, y su perniciosa influencia sobre la suerte de los grupos étnicos amazónicos (63). Stefano Varese produjo un notable estudio de caso, de las interrelaciones entre la sociedad Campa del Perú central y la sociedad peruana desie la Conquista (64). El tratamiento del destino de los nativos amazónicos por esta oleada de antropólogos culturales, se puede resumir en cuatro rubros: crítica de los prejuicios amazónicos; ejemplos de políticas y prácticas equivocadas; análisis del equilibrio ecológico que han logrado los grupos nativos con su medio: y finalmente recomendaciones para acciones más positivas (65).

Su crítica de los prejuicios amazónicos, se concentra en lo que ellos consideran dos prejuicios importantes de la sociedad nacional peruana frente a la porción selvítica del Perú: la creencia ingenua en la gran fertilidad de las tierras de la Amazonia, que supuestamente podría sostener grandes poblaciones: y la total incomprensión de los modos de producción indigenas. Argumentan que los nativos amazónicos han sido injustamente tildados de ociosos e incapaces para una actividad agrícola sostenida, porque no se entendió que su combinación Je caza, pesca y agricultura temporal constituyó una adaptación exitosa al bosque tropical. Los indígenas serranos que migraron a la selva, compartían algunos de estos prejujcios y adenás que, para su mentalidad agraria , toda tierra sin cultivar se ve como sin uso.

Los ajemplos de políticas y prácticas equivocadas de la sociedad nacional, con impacto negativo en la población indígena amazónica, son un rasgo común en estos escritos. Los ejemplos más recurrentes de dichas políticas son los siguientes:

- privilegios especiales para atraer colonos europeos en particular, frecuentemente con consecuencias discriminatorias para las poblaciones nativas.

- medidas para estimular la migración de campesinos serranos sin tierra a las zonas selváticas, que resultaron en ocupación de tierras de los nativos amazónicos y en relaciones generalmente malas entre nativos y migrantes. Entre estas medidas se mencionan, especialmente, la creación de haciendas en la sierra durante el siglo XIX, como consecuencia de las leyes liberales republicanas que ocasionaron la ruptura de las comunidades indígenas de la sierra; y aquellas que promovieron la colonización de la selva como alternativa a la reforma agraria en la sierra. 
- Prácticas y políticas de desarrollo caóticas e incoherentes, en relación a la explo. tación de recursos naturales. Se señalan especialmente diversas actividades extractivas, como el auge del caucho a fines del siglo pasado y comienzos del presente; el cual además de diezmar a la población nativa con enfermedades contagiosas, a veces causó la exterminación física planificada de los grupos indígenas.

Concesiones arbitrarias de tierras a misiones católicas y consorcios comerciales como la Peruvian Corporation en 1889. Las enormes concesiones a la Peruvian Corporation, que se comprometió a cambio a desarrollar la infraestructura de la zona y a promover la colonización con inmigrantes europeos (66); se iniciaron sin reconocer el hecho de que esta área albergaba a varios grupos nativos.

- Medidas para integrar a los grupos étnicos amazónicos a la nueva economía mo. netaria. Dicha integración, era especialmente consecuencia de actividades mercantiles extractivas necesitadas de mano de obra barata. Esta misma integración, se promovió también por los desarrollistas que veían la participación de los nativos en los mercados de producción y consumo como una forma superior de organización socioeconómica. En realidad, frecuentemente promovió la pauperización de los nativos.

- Migración selectiva por sexo, especialmente hacia la costa. Las mujeres que emigraban para trabajar como sirvientas redujeron la disponibilidad de mujeres casaderas, empujando a los hombres a dejar el grupo tribal, diluyendo así la cultura original (67).

- La imposición de sistemas foráneos de propiedad, por ejemplo las "cesiones" de tierras por la Peruvian Corporation en forma de lotes individuales, destruyendo así la estructura comunal de la tierra, característica de las comunidades nativas.

- La ausencia de una legislación especial para los grupos étnicos amazónicos. Con el advenimiento de la independencia el status especial que consagraba la inferioridad del indio desapareció, pero a la vez los elementos protectores de una legislación discriminatoria fueron eliminados también; lo cual condujo a una mayor explotación y más efectos negativos sobre la dinámica poblacional de los indios amazónicos.

Todas estas políticas, según estos autores del "indigenismo crítico", frecuentemente han ocasionado patrones demográficos desfavorables para las comunidades nativas; como la migración a las partes más inaccesibles de la selva; reducción del componente nativo en los asentamientos amazónicos - de situaciones en que constituian prácticamente el 100 por ciento de la población a menos del 50 por ciento; desintegración de las comunidades por la vía de la exogamia; a veces un aumento de la morbilidad, y una reducción del crecimiento poblacional de las comunidades amazónicas originales (68). La migración de los nativos a partes menos accesibles de la selva, parece haber sido parte integral de algunos planes de desarrollo. Un documento que analiza la migración espontánea hacia el valle del Apurímac, contiene el siguiente párrafo:

"Así, los planes de desarrollo futuro de la región ignoran intencionalmente a los nativos, sobre la presunción de que a medida que aumente la población y la fauna silvestre se haga menos abundante, los Campas se retirarán voluntariamente hacia el interior de la selva" (69).

El autor tiene cierto recelo respecto a esta política, pero parece aceptar esta consecuencia con una actitud hacia los derechos de los nativos que se ha caracterizado en el pasado por un desinterés común. Continúa: 
"La lógica de la premisa es discutible, pero es aparente que al menos en esta zona los Campas no son capaces, ni numérica ni tecnológicamente, de cuestionar seriamente el avance de la colonización".

Un punto importante que continuamente enfatizan los indigenistas, es el notable equilibrio ecológico que han logrado los grupos amazónicos nativos con su medio selvático $(70)$. Esto se contrasta con la supuesta falta de un equilibrio similar en los in. tentos actuales de desarrollo amazónico. Dice Chirif:

"El tipo de asentamiento semiconcentrados, o en algunos casos dispersos, junto con una economía de subsistencia, ha permitido la ocupación de la Amazonía sin riesgo para su ecología y por lo tanto para su sociedad. Esto no se cumple en el caso de la sociedad nacional, cuyos requerimientos económicos han impuesto un tipo de actividad negativa para la ecología y la sociedad amazónica... Los grupos tribales han podido construir sociedades autosuficientes, mientras que la sociedad nacional no ha podido desarrollar modos adecuados para colonizar esta región. Sólo ha podido crear núcleos artificiales con una economía dependiente; que mantienen a la mayoría de la población en condiciones marginales y favorece exclusivamente al pequeño sector del grupo dominante" (71). produjo:

El equilibrio original entre el medio ambiente y la organización social nativa

"un desarrollo armónico que se desintegró por factores externos, como la Conquista española y la agresión de la sociedad republicana" (72).

Al estudiar las condiciones actuales de los grupos tribales amazónicos y sus ajustes a los factores ecológicos, los indigenistas subrayan que es importante considerar este desequilibrio.

En algunos grupos del Fórum, había también una preocupación por los derechos de los nativos y las amenazas que surgen para ellos a partir de las políticas de colonización. La ley de Comunidades Nativas y Desarrollo Agropecuario de la Selva y Ceja de Selva (73), fue criticada por no ir suficientemente lejos en la protección de las comunidades nativas, y especialmente por negligir la provisión de las necesidades básicas de estas comunidades.

En un documento del Fórum se afirmaba lo siguiente:

"Las poblaciones y naciones indígenas del hemisferio occidental sufren todo tipo de genocidio, discriminación, esclavitud, peonaje, así como formas extremas de deprivación económica como son la desnutrición y sobrexplotación de la ma. no de obra. A estas técnicas de genocidio debemos agregar técnicas y procesos modernos como el control de la natalidad, esterilización, diversas formas de asimilación forzada; prácticas gubernamentales que ocasionan la destrucción.del medio ambiente y diversos programas de desarrollo que se presentan en nombre del progreso pero que tienen resultados perniciosos" (74).

Algunas personas en el Fórum resaltaron el efecto negativo de los esfuerzos de colonización sobre los grupos nativos, y opinaron que sería mucho mejor emplear a los grupos nativos y no migrantes serranos para ejecutar las políticas gubernamentales de colonización para las zonas amazónicas fronterizas.

\section{Ecologistas}

La preocupación por la ecología de la Amazonía (75) y su potencial limitado para sostener grandes poblaciones, es otra tendencia intelectual que en los últimos años ha introducido más prudencia en relación al desarrollo amazónico. Se pueden distinguir 
tres puntos de énfasis. Uno se dirige al potencial de recursos generales y agrícolas de la Amazonia, que se percibe como más limitado que lo que tradicionalmente se creía. Este énfasis comunmente integra resultados de estudio de suelos tropicales dentro y fuera de Sudamérica, mostrando la pobreza de muchos suelos tropicales, pese a la abundante vegetación y al delicado equilibrio establecido por el bosque tropical y su medio ambiente (76). Un segundo énfasis va hacia la perturbación del equilibrio tradicional con el medio, introducida por el aumento del crecimiento poblacional de las comunidades nativas amazónicas, debido a mejores condiciones de salud y mortalidad. Finalmente, la atención se dirige a la perturbación introducida por la migración de campesinos serranos a la Ceja de Selva. Esta migración, se dice, tiene dos efectos potencialmente perturbadores sobre la ecología tropical. Adenás de la mayor presión poblacional sobre el medio, esta migración frecuentemente se acompaña de la transferencia inadecuada a tierras tropicales de patrones y técnicas de cultivo desarrolladas en la sierra.

El último énfasis, está íntimamente ligado al punto de vista de los pesimistas de la colonización que se tratará más adelante.

La perspectiva ecológica era predominante en algunos de los mejores documentos presentados al Fórum. Uno de ellos da una descripción algo detallada del ecosistema amazónico, y enfatiza con fuerza sus límites para la colonización. Subraya que:

“... el suelo amazónico debe considerarse pobre y no apto para la agricultura.

De esta forma, sólo el 2.7 \% puede emplearse para la agricultura basada en árboles perennes, 46 o/o para madera y 38 o/o debe considerarse como bosques o áreas protegidas" (77).

Contrario al punto de vista de la Amazonía como solución a los problemas de población del Perú, el autor advierte que la migración espontánea de población de la sierra hacia la Ceja de Selva, crece ahora en proporción geométrica como resultado de la presión poblacional sobre las tierras disponibles en la sierra. Esto, afirma, es un factor importante en el deterioro ecológico de la Ceja de Selva. Critica por lo tanto de manera áspera la venta política, por ignorancia o demagogia, del "espejismo verde" de la selva peruana como medio para reducir la presión poblacional, el desempleo y la pobreza en la sierra y la costa. Opina que es precisamente esta actitud la que origina el conflicto entre la entrada irracional e incontrolada de grupos humanos y la ecología de la región; conflicto en el cual el gran perdedor es el país mismo.

Otro trabajo (78) en el mismo Fórum, describe como "mito detestable aquél que ha hecho creer a toda la nación que Amazonia es sinónimo de tierra fértil y fácilmente cultivable". El autor critica la "visión infantil" de que la selva puede ser la despensa del Perú; canto más peligroso que el de las sirenas y que sigue seduciendo a los peruanos. El autor opina que, especialmente en la fase actual del desarrollo amazónico, debe descartarse la idea que la selva puede transformarse en fuente significativa de producción alimentaria exportable a otras zonas del Perú. Muestra como la migración de campesinos de la sierra a la Ceja de Selva ha causado serios problemas de erosión por el uso creciente de métodos de "talar y quemar". Dichos métodos estaban en perfecta armonía con la ecología de la Ceja de Selva en un nivel mucho menor de densidad poblacional, pero se tornan crecientemente destructivos a medida que aumenta la población.

En un documento sobrc la importancia de la caza y la pesca en la provisión de proteínas para la población rural amazónica, el mismo autor señala que la mayor densidad poblacional ha disminuido la fauna en algunas áreas amazónicas (79). En otro momento menciona la enorme deforestación que se está llevando a cabo (80).

Una visión global de las diversas presiones negativas sobre la ecología amazónica peruana puede hallarse en un trabajo de Héctor Martínez, quien ha escrito frecuente- 
mente sobre la migración interna en el Perú. Entre las presiones especialmente desta: cadas están los rasgos mercantilista extractivos de la economía amazónica y la migración de campesinos serranos hacia la costa, que produce patrones de cultivo inapropiados para la ecología amazónica (81).

En el pasado, la necesidad del desarrollo amazónico se basa en requerimientos de bienestar de toda la población peruana. Sin embargo, muchos estudios recientes que abarcan la perspectiva ecologista arguyen que el desarrollo armónico sólo puede darse si es principalmente para sus habitantes actuales, y si la Amazonía no se ve como una colonia interna que responde principalmente a las necesidades de la población de là costa y la sierra. Dourojeanni dice:

"Una Amazonía peruana principalmente para el bienestar de los peruanos que viven ahí, o una Amazonía peruana principalmente para el bienestar de todos los peruanos. Estas parecen ser las dos alternativas básicss. Todo lo demás dependerá de cuál alternativa es aceptada. Hasta ahora, como los hechos (desafortunados según la descripción del autor) lo muestran innegablemente, la segunda alternativa ha dominado ampliamente" (82).

Recomendaciones semejantes para la política abundan en los trabajos de los ecologistas, e implican tácitamente al menos una actitud cauta frente a la inmigración rápida a la Amazonía.

Este punto de vista está penetrando lentamente en algunos círculos gubernamentales, según demuestra la siguiente declaración de un experto del gobiemo peruano, en un Taller sobre los efectos ecológicos de las crecientes actividades humanas en los ecosistemas del bosque tropical y sub-tropical:

"La intensidad de la explotación ha ido aumentando en los últimos affos, alcanzando proporciones alarmantes y causando la depredación de valiosos recursos que son esenciales para la economía tradicional" (83).

Este mismo punto ha sido bien documentado y resumido en diversos trabajos por Watters (84), quien hace una distinción entre sistemas estables e inestables de agricultura de tala y quema. Los sistemas estables, son antiquísimos sistemas tradicionales de ajuste racional al medio ambiente de bosque tropical, y siempre se acompañan de una baja densidad poblacional (85). Si la densidad poblacional aumenta, sea por menor mortalidad o migración -como es especialmente el caso del Perú con los campesinos serranos-dichosos sistemas pueden tornarse inestables. Las técricas de talar y quemar se harán disfuncionales porque, debido a la mayor densidad poblacional, los periodos de recuperación serán más cortos. Esto aumentará la competencia de la yerba mala y problemas de plagas. Por último, disminuirá la fertilidad del suelo y ocurrirá una seria erosión. En algunos casos, la mayor densidad poblacional puede promover la transición de cultivos alternos a nuevos sistemas de ajuste agrícola balanceado, basados en un patrón demográfico de mayor densidad poblacional y concentración de población; confirmando así la tesis de Boserup para estos casos. Sin embargo, en muchos casos dicha transición no ha ocurrido automáticamente y se ha dado más bien un serio deterioro ecológico (86). La recomendación de Watters en cuanto a política, es que esta transformación cultural debe iniciarse o acelerarse a través de un conjunto de medidas para contrarrestar el deterioro ecológico. Como recomendación adicional o alternativa sugiere deben buscarse formas de cultivos alternos que estén en armonía con la ecología, ahí donde no es viable un sistema de cultivo permanente.

Investigaciones recientes, que muestran que las técnicas tradicionales de talar y quemar pueden ser más eficientes que el método del bulldozer, aún para los cultivos contínuos, son otra advertencia contra la posibilidad de una rápida expansión agrícola a través de la mecanización de la Amazonía (87). 
La nueva perspectiva - la de tomar conciencia de la precariedad de los ecosistemas amazónicos y sus limitaciones para solucionar todos los problemas del Perú- aún parece limitada a pequeños grupos de expertos; al menos a juzgar por los editoriales de varios diarios limeños anticipándose al Fórum sobre la Amazonía. Por añadidura, la cantidad de investigación ecológica seria que se ha hecho sobre la Amazoníaperuana es insuficiente $y$, con algunas notables excepciones, mucho menos desarrollada que en el Brasil y otras áreas tropicales.

\section{Pesimistas de la Colonización}

Existen pocos estudios detallados sobre los fracasos y los éxitos de la colonización planificada de la Amazonia peruana (88). Aquellos que se han hecho no siempre están fácilmente disponibles. Los pocos estudios existentes difieren sustancialmente en calidad, metodología y comparabilidad.

Varían entre empresas científicas relativamente rigurosas y descripciones periodísticas más impresionistas. En conjunto, estos estudios llegan claramente a la conclusión que los esfuerzos planificados de colonización, que siempre han comprometido a grupos muy pequeños y por lo tanto han tenido poca o ninguna importancia demográfica en el Perú (89), han sido fracasos desoladores. Estos fracasos se concretan en las bajísimas proporciones de colonos que siguieron con el plan original de colonización (90). De los que no siguieron, algunos regresan a sus lugares de origen y otros permanecen en la zona pero no en la colonia original. Entre estos últimos, algunos se convierten en jornaleros agrícolas para los agricultores acomodados, mientras que otros se dedican al pequeño comercio u otras actividades no agrícolas. El fracaso se refleja también en los niveles de vida, a nivel de subsistencia, permanentemente bajos de los colonos, que definitivamente no son mejores que los de los migrantes espontáneos. Se hace referencia especialmente a programas de colonización auspiciados y sostenidos por el gobierno (91). Sin embargo, los planes privados o parcialmente privados han tenido problemas similares (92). Ádemás, las metas de colonización rara vez se alcanzan. Martínez da el ejemplo de un proyecto de colonización que después de seis años había establecido sólo a dieciocho colonos, de los 300 a 400 planificados (93). Entre las ra. zones por las que fracasan dichos esfuerzos, las más frecuentemente citadas en la lite. ratura (94), son las siguientes:

1. Objetivos confusos o erróneos. Esta es probablemente la rảzón más impor. tante por la que han sido tan decepcionantes los planes de colonización en el Perú. El ejemplo más saltante de un objetivo equivocado, es la colonización en lugar de reforna agraria en otras partes del Perú. Watters, que está a favor de la colonización, subraya que sólo puede ser una parte muy pequen̂́a de la solución a los problemas rurales y poblacionales de la sierra (95). Ocasionalmente, como ocurre en el programa de transmigración en Indonesia, los desastres naturales -como los terremotos-dan una razón por transferir gente a la selva.

2. Insuficiente claridad sobre las modalidades de los planes de colonización. Sin razón aparente, el énfasis de algunos planes está puesto en el desarrollo agrícola a través de parcelas individuales; en otros, está en formas cooperativas de explotación. En algunos casos, los planes cooperativos en relación a los servicios o la producción se impusieron sin un compromiso real de los participantes. El hecho que frecuentemente sólo los miembros de cooperativas pueden obtener crédito, infló el número de éstas. Además, para el caso de parcelas individuales, no existen criterios consistentes para determinar el tamaño óptimo del terreno, según las potencialidades del suelo. El modelo espacial del plan de colonización, con frecuencia se fija arbitrariamente. Sin racionalidad, algunos proyectos prefieren un plan lineal de colonización, mientras que otros 
revierten a una forma más concentrada. Los objetivos, como la expansión de la frontera agrícola y la creación de oportunidades de empleo, no siempre están bien coordinados; como se refleja con frecuencia en la introducción, sin planificación adecuada, de diversos tipos de agricultura mecanizada.

Ambas razones, objetivos confusos o erróneos, e insuficiente claridad sobre modalidades de planes de colonización, tienen su origen en la falta de una política coherente de colonización para la selva peruana. Martínez demuestra esto muy bien, al señalar cómo una serie de planes recientes de colonización tenian orígenes muy dispares (96).

3. Problemas con la adjudicación de las tierras (97). Los colonos a veces deben esperar durante largos períodos antes de tener títulos legales sobre la tierra. Como un título legal normalmente es requisito para obtener crédito, surgen otros problemas. Además, el sistema actual de adjudicación, más consistente con un sistema de agricultura continua, no siempre encaja bien con un sistema agrícola de talar y quemar. Los diversos procedimientos de adjudicación (venta pública, venta preferencial, venta con sorteo), no siempre se han empleado de manera consistente y bien engranada. El abandono del terreno adjudicado, en caso que llegue un nuevo ocupante, o si fracasa todo el plan de colonización, complica aún más todo el proceso.

4. La burocracia gubernamental ineficiente, que frecuentemente se aúna a un liderazgo inadecuado, y un rápido recambio de personal gubernamental. En un plan de colonización, los participantes debieron esperar cuatro meses antes que se identificaran concretamente las parcelas individuales (98). Estos incidentes con seguridad estimulan el proceso de deserción, antes de que la colonización realmente haya comen. zado.

5. Infraestructura y servicios inadecuados. La falta de puentes sobre ríos pequeños y la ausencia de un buen sistema de carreteras, hace muy difícil la comercialización de productos y el acceso a servicios; según Watters, se ha estimado que una colonización permanente nunca se da, si las unidades de explotación están a una distancia mayor de dos kilómetros de un camino (99). El factor de la accesibilidad física, con frecuencia se descuida al escoger los lugares de colonización. Martínez subraya que, en este sentido, la colonización planificada no se diferencia de la que se da espontáneamente (100). Las instalaciones para vivienda, salud y educación frecuentemente son inadecuadas, especialmente al inicio del proyecto, con lo que se favorece una tasa rápida de deserción. Los servicioș médicos, insuficientes en un contexto en que los migrantes están expuestos a nuevas enfermedades; la baja calidad de las escuelas y la distancia de éstas a las parcelas; todo juega un rol importante en este proceso. Las facilidades de crédito son confusas, complicadas, lentas, y no tienen continuidad. La asesoría técnica y servicios de extensión, muchas veces faltan, son insuficientes o de baja calidad (101).

6. Expectativas exageradamente optimistas, combinadas con promesas incumpilias sobre asesoría e instalaciones, posteriormente llevan a serias decepciones; especialmente si, como sucede con frecuencia, hay una baja en la productividad del suelo durante el segundo año. Esto, frecuentemente, trae como consecuencia que los colo. nos dejen la parcela original para buscar otra, reforzando un sistema inestable de agricultura de talar y quemar.

7. Conocimiento insuficiente del suelo destinado a la colonización. Los planes de colonización, frecuentemente se han promovido sin ninguna consideración por los diferentes tipos de suelos y sus posibilidades de explotación agrícola. La mayoría de los 
planes se han iniciado sin ningún estudio real de la calidad del suelo. Aún donde sí se hicieron dichos estudios, a menudo fueron demasiado generales para guiar una adecuada microplanificación.

8. Preparación insuficiente de los participantes para la colonización en general y para las modalidades específicas del plan. En un plan de colonización, se encontró que sólo algo más del 30 o/o eran agricultores activos antes de inscribirse en el plan (102). Los colonos que participan en planes dirigidos a la producción para el mercado, frecuentemente vuelven a los cultivos de subsistencia porque carecen de experiencia en cultivos para el mercado. No parece haber procedimiento de selección alguno para eliminar a los colonos dudosos y así reducir el índice de deserción. El contexto geográfico del que proceden los colonos también afecta su rendimiento posterior. Los que vienen de la sierra, parecen tener los problemas más difíciles debido a su deseo de recrear una agricultura de tipo serrano en un medio muy diferente. Además, el contexto geográfico muy dispar de los colonos de planes oficiales, en contraste con los migrantes espontáneos, frecuentemente ha creado más problemas.

9. Prácticamente no se han hecho estudios rigurosos de costo-beneficio de los planes de colonización en el Perú. Sin duda dichos estudios son difíciles de realizar, por el tremendo problema de obtener información correcta y de comparar categorias de costos. Sin embargo, varios autores han señalado los altos costos de estos planes y el largo periodo para el que requieren financiamiento. Watters, por ejemplo, señala que en un plan de colonización aparentemente exitoso, compuesto originalmente de migrantes de una barriada limeña, el gobierno y otras agencias habian hecho aportes sustanciosos, mucho mayores que el costo inicial de la colonización. La introducción indebida de la mecanización, muchas veces ha incrementado sustancialmente los costos y reducido la tasa de ganancia. La tasa de recuperación del crédito obtenido frecuentemente es baja. El único estudio que ha encontrado el autor, y que hace un serio esfuerzo por estudiar los costos de un plan de colonización, es del que se ubica en Tíngo María. Aunque el estudio señala las limitaciones de los datos - limitaciones que probablemente conducen a subestimar los costos - se encontró que el costo por familia establecido era de más de $\$ 10,000$ (104).

Aunque la falta de una política coherente de colonización se señaló como una importante razón del fracaso, se han hecho esfuerzos esporádicos de establecer una política más coherente para la colonización de la selva. El ejemplo más saltante es un interesante documento de CENCIRA, de hace algunos años (105). Este documento, que liace amplias referencias a varios planes fracasados de colonización, es muy prudencialista en su tono y trata de desarrollar una política de colonización para la selva, que éncaje mejor con las políticas económicas generales y las de desarrollo rural en el Perú. Subraya enfáticamente el modesto papel que puede cumplir la selva en dichas políti. cas. Los documentos aunque no descartan la migración continua hacia la selva, nos recuerdan que:

"La selva no debe ser vista como tierra prametida por la población de otras regiones del país, ni como una despensa nacional" (106).

El documento subraya que: "La expansión de la frontera agrícola debe basarse, fundamentalmente, en la plena incorporación de las tierras no explotadas, de la costa y la sierra. En vista de la escasez relativa de tierras en explotación en la selva, la expansión de la frontera agrícola en la zona de la selva, tiene que dirigirse primeramente a las necesidades de los campesinos locales, y sólo en segundo término y en casos especiales a las necesidades de otras regiones" (107).

El documento además considera que: "La colonización organizada por el Estado, no debe seguir siendo la mejor forma de extender la frontera agrícola del país". Estas 
consideraciones sobre la colonización planificada en la selva, deben equilibrarse con información sobre la colonización espontánea, acerca de la cual la ignorancia probablemente es aún mayor (108). Aunque siempre ha habido alguna migración hacia la selva, ta migración espontánea sustancial de campesinos serranos a la selva, empezó hace treinta afios (109). El crecimiento poblacional en la selva ha sido más rápido que el promedio nacional, en el periodo de 1940 a la fecha (110) En términos absolutos, sin embargo, es pequefio debido a la población base reducida.

Aunque no se han hecho estudios reales sobre la motivación de los migrantes espontáneos, Watters afirma que las condiciones de pobreza de la sierra, mucho más que la atracción de la selva, ocasionan la migración de campesinos serranos a la selva (111). Los migrantes espontáneos, sin embargo, en contraste con los miembros de planes de colonización, parecen estar más contentos con su destino en su nueva localidad que en su lugar de origen en la sierra (112). La tasa de deserción de los migrantes espontáneos, es notablemente más baja que la de los participantes en planes de colonización (113). El mejor éxito de la migración espontánea, según Aramburú, se debe a une mayor cohesión grupal y al hecho de que, en estos grupos, fue mayor el énfasis en cultivos tradicionales que, aún con la baja rentabilidad comercial, permitían cubrir las necesidades de alimentación de la familia (114). Por otro lado, en varlos planes de colonización el énfasis se pone en la producción para el mercada, con la consecuencia de que los alimentos para el consumo deben comprarse a crédito, debido al ingreso insuficiente por el cultivo comercial (115).

Aunque pocos prudencialistas considerarian una mayor ocupación de la selva como solución al rápido crecimiento poblacional y otros problemas sociales o económicos, el deseo de ver por lo menos un incremento moderado de asentamientos humanos en la selva, seguirá siendo un rasgo de las políticas de población explícitas e implícitas en el Perú. Entre las recomendaciones de un grupo de peruanos destacados, que recientemente se reunió para discutir los problemas nacionales de población, podemos leer lo siguiente: "para aumentar la producción alimentaria... la frontera agricola necesita expandirse a través de pequeños y medianos proyectos de migración, la recuperación de suelos y los asentamientos rurales en la Selva y Ceja de Selva" (116).

En el mismo evento nacional sobre población (Tarma 1979), un conocido experto peruano en alimentación del INP subrayó que "es indispensable continuar la expan. sión de tierras a través de nuevas obras de irrigación, así como asentamientos rurales en la Selva y Ceja de Selva" (117). Sin embargo, para que dicha expansión sea rentable y permanente, debe darse prioridad a las inversiones para conservar y aumentar la productividad de la tierra. Marc Dourojeanni, quien ha escrito frecuentemente sobre el precario equilibrio de la ecología amazónica, y ha señalado una advertencia en contra de las expectativas utópicas comunmente existentes en el Perú acerca de las posibilidades de desarrollo de la amazonía peruana, también ha opinado a menudo en favor de políticas de desarrollo de acuerdo al precario medio ambiente amazónico.(118).

\section{CONCLUSION}

No hay duda que las políticas amazónicas del Perú se orientarán hacia el desarrollo amazónico. La perspectiva emerge claramente en la nueva Constitución peruana aprobada en 1979 (119). 
Con excepción del artículo que afirma que Lima es la capital del Perú, la nueva Constitución no menciona ninguna área geográfica en particular, salvo la Ámazonía. El artículo 120 dice:

"El Estado promoverá el desarrollo de la Amazonía. Dará a esta región trato especial cuando sea necesario. Se encargará a una institución técnica y autónoma el inventario, la investigación y la evaluación de los recursos naturales de esta región" (120).

El propósito de este trabajo no es desalentar al Perú de desarrollar su selva. Pero opinamos que ésto debe hacerse con un conocimiento mucho mejor de sus recursos básicos y su sistema ecológico. Un mejor conocimiento de las diversas ideologías en relación al desarrollo amazónico es, además, una condición sine que non para llegar a políticas más inteligentes.

En el debate amazónico, debe prestarse mucha más atención a distinguir los hechos de la fábula, y a revisar el grado en que las diversas ideologías del desarrollo amazónico coinciden con los hechos. Esto, junto cón el deseo de que se ponga más énfasis en las perspectivas prudencialistas, que hasta la fecha han tenido un impacto insuficiente en las políticas amazónicas peruanas, ha sido el objetivo de este trabajo.

(Traducción de Rocio Palomino) 


\section{NOTAS}

1. Héctur Vargas Haya, Amazonía: Realidad o Mito. El Reto de la Integración Amaz6. nica (Lima, 1977), p. 95 y Compañía Editora Nacional, Moderno Atlas Ilustrado (Sao Paulo, 1977) p. 17. Se emplean diferentes criterios para definir la Amazonía y las diversas partes amazónicas de Sudamérica. Por lo tanto, pueden encontrarse en la literatura cifras muy diferentes. Por ejemplo, Charles, Wagley, en la introducción a Man in the Amazon (Gainesville, 1974), p. 22 afirma que "el área amazónica brasilera ocupa dos tercios del área total del Brasil". Para el caso del Perú, también pueden hallarse en la literatura cifras superiores al sesenta por ciento. Ver por ejemplo Edgardo Mercado Jarrín, "Pacto Amaź́nico: Nuevo Esquema Geopolftico", Estudios Geopolíticos y Es. tratégicos I: 4 (Mayo 1980): 65 .

2. Forum: "Amazonía, Conquista del Presente". Lima y Pucallpa, Noviembre 6.9, 1978. En aras a la brevedad, se empleará el término Fórum en este trabajo para referirse a las ponencias presentadas en el Fórum sobre la Amazonía. Todas las ponencias presentadas al Fórum sobre la Amazonía se distribuyeron en copias mimeografiadas al inicio del evento. Todas las referencias en este trabajo corresponden a dichas ponencias mimeografiadas. Como en muchos otros países latinoamericanos, especialmente en comparación con países anglosajones, los arquitectos y sus asociaciones tienen un rol más preponderante en la vida política y sus intereses abarcan un campo más amplio de problemas. Una demostración de esto es que en 1977 el colegio de Arquitectos organizó un Fórum sobre Lima en el año 2,000 con varios enfoques de población.

3. Hay bastantes diferencias entre diversos autores en relación a la altura aproximada donde comienza la Ceja de Selva. Por ejemplo, en el famoso Atlas Geosáfico de los Padsajes Peruanos (Lina, 1969) el director del proyecto, Carlos Peñaherrera del Aguila (p. 174), considera que comienza a los 2,000 metros; mientras que Javier Pulgar Vidal, en el mismo Atlas (p. 114), la sitúa a 1,000 metros. Marc Dourojeanni en "Una Nueva Es. trategia para el Desarrollo de la Amazonía Peruana", Revista Forestal del Ponú 6: 1-2, $(1975$ - 1976): 42, fija su límite promedio superior en 3,800 metros y su límite inferior en 600 metros. Para fines jurídicos de la Reforma Agraria, la Ceja de Selva tuvo una definición muy detallada. Ver CENCIRA, "Recomendaciones Generales para una Política de Colonización en la Selva" (Lima, 1974). Mimeografiado, pp. 76-78.

4. El Dr. Alberto Chirif me hizo reparar en este hecho.

6. Para una descripción detallada de ambos ecosistemas y su impacto cultural en la pobla. ción prehispánica, ver Betty J. Meggers, Amazonía. Man and Culture in a Counterfeit Paradise (Arlington Heights, 1974) y Betty J. Meggers, "Environment and Culture in Amazonfa" en Charles Wagley, ed., Man in the Amazon (Gainesville, 1974), pp. 99-110. Para una visión diferente de la demografía e interrelación con la ecología ver Donald Lathrap, "The Hunting Economies of the Tropical Forest zone of South America. An attempt at Historical Perspective" en Daniel R. Gross, ed., Peoples and Cultures of Native South America (New York, 1973), pp. 83-95 y Robert L. Carneire, "Slash and -Bum Cultivation among the Kutiuru and its Implications for Cultural Development in the Amazon Basin", en Daniel R. Gross, ed., Peoples and Cultures of Native South America (New York, 1973), pp. 98-123. Ambos autores, por supuesto, reconocen las diferencias ecológicas entre estos dos medios y las toman en cuenta en sus interpretaciones. Lathrap estima que los grupos tropicales que viven lejos de la llanura inundablc no deben considerarse como adaptaciones originales a sus ecosistemas sino como adaptaciones forzadas de sociedades agrícolas más desarrolladas que tuvieron que dejar su medio ambiente ribereño. Carmeño enfatiza que Meggers subestima la productividad del bosque agrícola, que las aldeas rara vez crecen hasta que presionan la capacidad de la tierra y que por tanto los otros mecanismos que afectan la dinámica poblacional son operativos. 
6. Fðrum. Emilio Romero, "El Hombre en la Amazonía Peruana", p. 14.

7. Fórum. Paul Macia Morey, "Hacia una Nueva Política para el Morador", p. 2.

8. José Carlos Mariátegui, Slete Ensayos de Interpretaclón de la Roalidad Peruana (Lima, 1968) pp, 154-180.

9. Para una visión panorámica más completa de estos incentivos ver Alberto Chirif, "Ocupación Territorial de la Amazonía y marginación de la Población Nativa", Amúrica Indícena 35: 2 (Abril-Junio 1975): 271-275. Para una visión panorámica de la legislación para promover la colonización de la Selva ver Héctor Martínez, Las Colonizaciones Selváticas Dirigidas en el Perú. Antecedentes, actualidad y perspectivas (Lima, 1976), pp. 53-64.

10. CENCIRA, Recomendaciones Generales para una Política de la Colonización en la Selva (Lima, 1974). Mimeografiado.

11. Martin T. Katymann, Cities and Frontiers in Brazil (Cambridge 1977), pp. 4,79.

12.J. M. Hardjone, Transmigration in Indonesia (Jakarta, 1977).

13. Ver, entre otros: Carlos Aramburú, "Las Migraciones a las zonas de Colonización en la Selva Peruana: Perspectivas y Avances", Debates en Antropologia 4(1979): 87, y Ro. ger Rumrill y Pierre de Zutter, Amazonía y Capitalismo, Los Condenados de la Selva (Lima), P. 29 y Alejandro Portes, "Migration and Underdevelopment in Politics and Society", Politics and Society 8: I (1978): 32, 25-27.

14.James Petras y Robert La Porte, Perú: Transformación Revolucionaria o Modernización (Buenos Aires, sin fecha), p. 31.

15. La Carretera Marginal se considera como parte de un plan para proveer al Perú de tres carreteras longitudinales paralelas (a lo largo de la costa, que está terminada, a través de la Sierra y a través de la Selva, ambas sin concluir) conectadas con diversos caminos de penetración transversales (Robert Manetl, Perú (Londres, 1969), p, 231). Para el entorno político y financiero de las políticas de construcción de carreteras de Belaúnde Terry ver Pedro Pablo Kuczynski, Peruvian Democracy under Economic Stress. An account of the Belaúnde administration 1963-1968 (Princeton, 1977), especialmente pp. 52-59.

16. Ver por ejemplo su discurso inaugural en el Seminario Nacional sobre Población y De* sarrollo en el Centro de Estudios de Población y Desarrollo, Primer Seminario de Población y Desarrollo (Paracas, 1965), p. XXXIII, y diversos pasajes de su libro La Conquis* ta del Perú por los Peruanos (Lima, 1969).

17. John Strasma, "The United States and Arrarian Reform in Peru" en Daniel A. Sharp, ed., U.S. Foreign Policy and Peru (Austin, 1972), p. 181.

18. Naciones Unidas, Peru's National Experience in the Formulation and Implementation of Population Policy (Nueva York, 1978), pp. 6.9.

19. Juan J. Wicht, La Situación Demográfica en el Perú, AMIDEP, Reunión Nacional sobre Población (Tarma, junio 1979).

20. Amazonía, Desarrollo y la Marginal. La Prensa, 10 de marzo de 1979. Debe anotarse, sin embargo, que la propuesta de plan de gobierno 1980.1985 del partido de Belaúnde, Acción Popular, recomienda explícitamente $\mathrm{l}_{2}$ inclusión de los principios de política de población en el Plan de Salud (Acción Popular, Propuesta de Lineamientos del Plan de Gobierno 1980-1985, p. 27). El actual Gobierno de Belaúnde (1980) planea buscar una política activa de población, de la cual es componente importante la reducción de la tasa de crecimiento poblacional.

21. El Comereio, 28 de agostu de 1980.

22. El Comereio, 10 y 11 de agosto de 1980.

23. Nuevos Criterios para Colonizar la Selva, La Prena, 21 de agosto de 1980.

24. Héctor Vargas Haya, Amazoní Realidad o Mito, El Reto de la Integración Amazónica (Lima, 1977), p. 59.

25. Héctor Vargas Haya, Amazoní Realldad o Mito, El Reto de la Integración Amazó. nica (Lima, 1977), p. 89. 
26.Héctor Vargas Haya, "Amazonia: Gigante Dormido" en La Prensa 10 de marzo de 1979.

27. Héctor Vargas Haya, Amuzonín Realldad o Mto. El Reto de la Integración Amazónica (Lima, 1977), p. 93.

28. Ministerio de Vivienda: Dirección General de Desarrollo Urbano, Plan Nacional de Desarrollo Urbano. Volúmen 8. Flujos Migratorios (Lima, 1973), p. 12.

29. Héctor Vargas Haya, Amazonf́ Realidad o Mtto. El Reto de la Integración Amazónica (Lima, 1977), p. 35. Si se sopesa ésta afirmación con la descripción de la riquera de los recursos naturales amazónicos, no se puede sino considerar que el autor es inconsistente.

30.Héctor Vargas Haya; Amazonia Realidad o Młto. El Reto de la Integración Amazónica (Lima, 1977), p. 81.

31. Oiga, Número 93, 1979 y El Comercio, 10 de octubre de 1979.

32. Hernán Alva Orlandini, Un Alto en el Camino del Perú, Crítica y Consenso. Vol. I (Lime, 1980). pp. 335-338.

33. Para una visión panorámica del expansionismo brasilero y la geopolítica amazónica ver Lewis A. Tambs. "Geopolities of the Amason" en Charles Wagley, ed., Man in the Amazon (Gainesville 1974) pp. 45-87. El artículo demuestra que la penetración pe. ruana en la Amazonia no siempre puede describirse como pasiva y reactiva. Ver también José Zárate Lescano, "Perú y Brasil": Antecedentes Limitrofes", Zatudios Goopolíticos y Estratécicos 3 (1979): $89-94$ y 4 (1980): 122-125.

34.Centro de Altos Estudios Militares. Colonización y Desarrollo Económico de la Selva Central (Lima, 1958) Mimeografiado.

35. Oficina Nacional de Estadísticas y Censo, Año Mundial de la Población: Actividades Internacionales y Nacionales. La Política de Población del Perú y la Conferencia Mundial de Población. Parte II. Boletín Especial Nro, 3 (Lima, 1974), p. 4.

86. República Peruana - Instituto Nacional de Planificación, Plan Nacional de Desarrollo para 1977 y 1978. Plan Global (Lima, 1977), pp. 39-40.

87. República Peruana, Presidencia de 12 República. Instituto Nacional de Planificación, Plan Nacional de Desarrollo para 1979-1980. Plan Global (Lima, 1979), pp. 57-66.

38. Ministerio de Educación, Seminario-Taller de Promoción de Servicios Integrados en Zonas de Frontera (Lima, 1979). Mimeografiado.

39.Para mayor información sobre el drea amazónica en el pensamiento geopolítico brasileño ver John Child, "Geopolitical Thinking in Latin America". Latin Amoxionn Reaearch Revlew 142 (1979): 89-112. El mismo axtículo contiene algunas referencias al Perú. Aunque es cierto que el peasamiento geopolítico es más preponderante en el Brasil, el autor subestima su papel en el Perú.

40.Habia una delegación de la Embajada Brasileña en el Perú asistiendo al Forum, y uno de aus miembros presentó una visión penorámica de la experiencia amazónica brasileña. Ver Fórum. Manuel Emilio Gelia, "Brasil: Experiencia Amazónica".

41. Fórum. Eráclides Vergaray, Tratado Amazónico.

42. Förum. Recomendaclones.

43.Crónica Institucional, "Primer Seminario de Geopolítica y Estrategia", Eutudios Geopolrtie as y Iatratédeos I (Enero-Marzo 1979).

44. Edgardo Mercado Jarrin, "Proyecciones del Brasil", Estudlos Geopolíticos y Estrat6is acos 3 (Diciembre 1979): 5-14.

18.Víctor Miró Quesada Ureta, "El Mar y los Ríos. ¿Es el Perú un País Bioceánico?", Eatudios Geopolítieos s Entratócicos 3 (Diciembre 1979): 30-33.

46. Edgardo Mercado Jarrín, "Pacto Amaxónico: Nuevo Esquems Geopolítico", Eatudios Geopolíticoa y Entratégicos 4 (Mayo 1980): 62 .

47.Edgardo Mercado Jarrín, "Pacto Amazónico: Nuevo Esquema Geopolítico", Eatudios Geopolítieon I Eatrat Geloos 4 (Mayo 1980): 66, 
48. Virgilio Roel, La Crisis General del Capitallamo y de la Economía Peruana (Lima, 1978), p. 19. Como en varios otros escritos, Roel emplea a veces términos muy ambiguos. Los términos "desertificación relativa" y "desertificación demográfica" seguramente confundirán a algunos lectores.

49. Emilio Castañón Pasquel, "Población y Recursos", Estudios Geopolíticos y. Estratedeos 3 (Diciembre 1979): 117.

50. Edgardo Mercado Jarrín, "El Desarrollo del 'Hinterland'. La Selva Central: Dilatado Vacío Espacial", Estudios Geopolíticos y Estratégleos 4 (Mayo 1980): 21.

51. "Alarma en la Frontera", Caretas (27 de agosto de 1979): 38-39.

52. Héctor Vargas Haya, Amazonia. Realidad o Mito. El Reto de la Integración Amazónica (Lima, 1977).

53. Este plan fue promovido en 1948 por un político argentino, Gabriel del Mazo.

54. Fórum: Hernando Quintana Arriola. "El Trópico - La Nueva Frontera de los Asentamientos en América Latina", p. 7.

55. Ver su libro Amazonía, Realidad o Mito. El Reto de la Integración Amazónica (Lima, 1977). Aunque su libro contiene ocasionalmente información útil, en conjunto es un tratado superficial de la Amazonía Peruana. Se enmarca en una confianza ingenua en la tecnología para la resolución de muchos problemas del desarrollo amazónico, y sus recomendaciones incluyen grandiosos proyectos tecnológicos. Sin embargo, el autor merece reconocimiento en relación a dos puntos.

Aunque su utilización de la investigación es muy dispareja, parcial y en general superficial, resalta la necesidad de más investigación amazónica en el Perú. En segundo lugar, en momentos en que los intereses nacionalistas estrechos parecen haberse desarrollado en diversas partes de Sudamérica, su énfasis en los esfuerzos de cooperación de los países interamazónicos para desarrollar la Amazonía es un cambio bienvenido en el tema de muchos debates actuales sobre asuntos internacionales en Sudamérica,

56. Héctor Vargas Haya, Amazonia Realidad o Mito. El Reto de la Integración Amazónica (Lima, 1977), p. 66.

57. Para una breve referencia a algunos de estos fracasos, ver Robert Manett, Perú (Londres, 1969), p. 232 y R.J. Owens, Peru (Londres, 1964), p. 13. Se puede encontrar información más detallada en Robert C. Eidt, "Pioneer Sttlement in Eastern Peru", Annals of the Association of American Geographeis 52 (1962): 255-278.

58. Ver Naciones Unidas Perú. National Experience in the Formulation and Implementa. tion of Population Policy, 1960-1976 (New York, 1978), p. 35.

59.El Movimiento Indigenista es una importante corriente intelectual del pensamiento social peruano, y normalmente se ha preocupado más de los indigenas serranos. Para una visión global, buena y precisa de los diferentes tipos de pensamiento indigenista en el Perú y de algunos aspectos históricos y raíces culturales de éste, ver Manuel M. Marzal, "Indigenismo y Constitución", Shupthui 8 (1978): 34-53.

60. Es interesante señalar que la nostalgia del Tahuantinsuyo, frecuentemente basada en una visión muy idealizada de la sociedad inca, se emplea a veces a favor de la opción opuesta, la convivencia de un vigoroso desarrollo amazónico. Ver por ejemplo Fernando Be. laúnde Terry, "Amazonía, Desarrollo y la Marginal", en La Prensa, 10 de marzo de 1979.

61.Para una visión global del impacto de las políticas de desarrollo amazónico del Brasil, ver Shelton H. Davis, Victims of the Miracle. Development of the Indians of Brazll (Cambridge 1978), y Fernando H. Cardoso y G. Muller, Amazonía. Exparsáo do Capitalismo (Sao Paulo, sin fecha).

62. Manuel M. Marzal, "Indigenismo y Constitución", Shupihui No, 8 (1978) 44-47.

63. Alberto Chirif, "Ocupación Territorial de la Amazonía y Marginación de la Población Activa", América Ind fgena 35: 2 (Abril-Junio 1975): 265-295.

64. Ste fano Varese, La Sal de los Cerros. Una aproximación al mundo Campa. (Lima, 1973).

65. Este resumen se basa en los siguientes artículos y libros:

- Ramón Aranda de los Ríos, Marankiari, Una Comunidad de la Selva Peruana (Lima, 1978). Mimeografiado.

- Alberto Chirif, ed, Comunidades Nativas de Selva Central: Diagnóstico Socloeco. nómico (Lima, 1975). Mimeografiado. 
Alberto Chirif, ed., Etnleidad y Ecologia (Lima, 1979).

- Alberto Chirif, "Ocupación Territorial de la Amazonia y Marginación de la Pobla. ción Activa", América Indígens 252 (Abril-Junio 1975) pp. 265-295.

- Alberto Chirif, La Cuestión de las Tierras y del Desarrollo Económico de las Comu. nidades Nativa. Despojo y Reivindicación (Lima, 1978). Mimeografiado, pp. 26-42.

- Richard Smith, Los Amuesha, una minorin amenazada en Comunidados Indigenas en Desalojo y Reivindicación (Lima, 1978) Mimeografiado, pp. 1-25.

- Ricardo Falla Barreda, Observaciones Preliminares sobre la Cuenca del Río Negro. Satipo (La Molina, 1979). Mimeografiado.

- Stefano Varese, La Sal de los Cerros. Una aproximación al mundo Campa (Lima, 1973).

En las paginas siguientes, las 'referencias arriba anotadas sólo se repetirán en caso de cita directa o cuando sea necesario por razón de claridad. Ocasionalmente se citarán otras referencias en el texto.

66. La mayor parte de las obligaciones contractuales nunca se pusieron en efecto. La Compañía se limitó a la explotación agrícola de tipo hacienda, con énfasis en el café. Ver Ramón Aranda de los Ríos. Marankiari. Una Comunidad de la Selva Peruana (Lima, 1978). Mimeografiado.

67. Richard Smith. Las Muchachas Indias son las Mejores Domésticas. Otro aspecto de Et nocidio en Comunidades Nativas en Despojo y Reivindicación (Lima, 1978). Mimeo. grafiado, pp. $43-48$ y Stefano Varese, La Sal de los Cerros. Una aproximación al mundo Campa (Lima, 1973), p. 269.

68. La demografía de los grupos étnicos originales de la Amazonía apenas se ha estudiado. Para un esfuerzo reciente, en estimaciones gruesas de número y crecimiento poblacio. nal, ver Luis M. Uriarte, "Poblaciones Nativas de la Amazonía Peruana", Ammzonía I: 1 (1976): 9.58.

Puede hallarse información interesante sobre su distribución poblacional en Stefano Varese, La Sal de los Cerros. Una aproxinación al mundo Campa (Lima, 1973): 341 360. Apéndice 1: Sociedades de la Selva y Situación Política. Una extensa descripción de la demografía de las Comunidades Indígenas en la Selva Central, puede hallarse en Alberto Chirif, e.a. Comunidades Nativas, Selva Central: Diąnóstico Socioeconómico (Lima, 1975). Mimeografiado.

69. Norman R. Stewart, "Migration and sttlement in the Peruvian Montaña: The Apurímac Valley", The Geographical Review 45: 3 (Abril 1965): 147.

70. Varios legos e incluso algunos antropólogos presentan una figura muy simplista de los sistemas de talar y quemar y de su relación con la densidad poblacional y el desarrollo cultural. La dinámica poblacional pre-ibérica de los nativos amazónicos es tema de acalorados debates entre antropólogos culturales que conocen la zona. Mientras Meggers sostiene una teoría ecológica estrecha, Carneiro presenta una teoría más amplia. Según Meggers el grado relativamente bajo de desarrollo cultural y las bajas densidades pobla. cionales de las comunidades amazónicas eran consecuencia directa de la pobreza del suelo amazónico, Carneiro, por el contrario, considera que los asentamientos amazónicos no realizaron totalmente su potencial agrícola con métodos de talar y quemar, y por lo tanto podrían haber tenido poblaciones mayores. Los estímulos económicos y políticos que estuvieron ausentes en la Amazonía habrían sido necesarios para obtener densidades poblacionales más al tas. Ver la colección de artículos en Daniel R. Gross, ed., Peoples and Cultures of Native South America (N. York, 1973) y Betty J. Meggers, Amazonia Man and Culture in a Counterfert Paradise (Arlington Heights, 1974). Para una visión panorámica muy interesante de los diversos medios amazónicos y sus implicancias respecto de las densidades poblacionales, ver William $M$. Denevan, ed, the Native Population of the Americas in 1942 (Madison, 1976), pp. 205-233.

Sus estimados, basados en extrapoblaciones de densidades conocidas para un grupo en una área física o cultural conocida (método de habitat-densidad), son mayores que muchos estimados previos para la gran Amazonía. Sin embargo, sus cifras pueden ser criticadas. Además de debilidades reconocidas por el propio autor, éste asume implícitamente que las poblaciones tienden a aproximarse al límite de la capacidad del medio am. biente. El autor reconoce la posible validez del "efecto de colchón" que defiende Thomas P. Myers en "Defended Territories and no man's lands" American Anthropologiot 78 (Junio 1976): 354-55, quien enfatiza que una parte sustancial de la Amazonía no fue ocupada debido al deseo de grupos vecinos hostiles de mantener zonas amplias y relativamente vacias que sirvieran de "colchón". 
71. Alberto Chirif, "Ocupación Territorial de la Amazonía y Marginación de la Población Activa", Amórica Indígena 25:2 (Abril-Junio 1975): 266-267.

72. Alberto Chirif, La Cuestión de las Tierras y del Desarrollo Economico de las Comunidades Natives on Comunidades Nativas. Despojo y Reivindieación (Lima, 1978). Mimeo. grafiado, p. 32 y Stefano Varese, La Sal do los Cerros. Una Aproximación al mundo Campa (Lima, 1973), p. 55 y p. 67.

73. La primera versión de esta ley apareció en 1974. Una versión revisada apareció en 1978. Para una discusión de estas leyes ver Carlos Mora y Alberto Chirif, "Comentario sobre la Nueva Ley de Comunidades Nativas (D.L. 22175)", Shupthui No. 8 (1978): 12.14 y Manuel García Rendueles, "Ley de Comunidades Nativas y de Desarrollo Agropecuario de la Selva y Ceja de Selva (D.L. 22175)". Shupihui No. 8 (1978): 15-29.

74. Fórum. Manases Fernández Lancho. "Situación Económica, Jurídica y Cultural de los Pueblos Indigenas de la Hilfa", p, 3.

75. Para una buena descripción del ecosistema de la Amazonía (excluyendo la Ceja de Sel. va) ver Betty J. Meggers, Amazonia Man and Cultuxo in Counterfeit Paradise, (Arlington Heights, 197), pp. 6-38.

76. Adernás de sus propios aportes en varios artículos, Marc Dourojeani resume los resultados de estos estudios, Ver especialmente Marc J. Dourojeanni, "Una Nueva Estrategia para el Desarrollo de la Amazonía Peruana", Revista Forestal del Peru. 6:1-12 (1975. 1976): 41.58 .

77. Fórum. Jorge Mailleux, "La Ecología y los Recursos Naturales en la Integración y Desarrollo de la Amazonía Peruana", p. 1.

78. Fórum, Marc J. Dourojeanni, "La Revolución y una Nueva Estrategia para el Desarrollo de la Amazonia Peruana", p. 2.

79.Paul V. Pierret, y Marc J. Dourojeanni, "Importancia de la caza para Alimentación Humana en el Curso Inferior del Río Ucayali, Perú", Revista Foreatel del Perú, 1:2 (Octubre 1967): 10-21.

80. Marc J, Dourojeanni, Utilización de los Recursos Naturales Renovables en el Perú. Mesa Redonda No, 1. Anales de CONIAP, pp. 55-66. Referencia incompleta en reimpresión.

81, Héctor Martínez, "El Saqueo y la Destrucción de Ecosistemas Selváticos del Perú", Amazonía Peruana 1: 2 (1977): 7-28.

82. Marc J. Dourojeanni, "Una Nueva Estrategia para el Desarrollo de la Amazonía Perua. na", Revista Forestal del Perá 6: 1-2 (1975-1976): 51.

83.UNESCO. Programme on Man and the Biosphere. International Working group on Project 1: Ecological aspects of increasing human activities on tropical subtropical forest ecosystems. Final Report Rio de Janeiro 11-15. Feb. 1974 (Paris, 1974), p. 75.

84. Ver R.F. Watters, "The Shifting Cultivation Problems in the American Tropics", Reunión Internacional sobre Problemas de Agricultura en los Trópicos Húmedos de América Latina (Lima y Belem Do Para, 1966). R.F. Watters, "Shifting Agriculture Its Past, Present and Future". The use of Ecological Guidelines for Development in the American Huimid Tropics and Proceeding (Caracas, 1974). International Union for Conservation of Nature and Natural Resources, 1975.

R.F. Watters, La Agatcultura Migratoria on el Perú (Mérida, Venezuela, 1968). Mimeografiado.

85. No slempre está claro, en los escritos sobre este tema, si los autores consideran la baja densidad poblacional como la causa del sistema de talar y quemar o si la baja densidad poblacional es una consecuencia directa de los sistemas en uso de talar y quemar. El segundo punto de vista debe prevalecer cuando se trata de las comunidades nativas originales de la Amazonía, Una causalidad en dirección opuesta es más apropiada cuando se considera la presión reciente que ejerce el crecimiento poblacional sobre el sistema de talar y quemar.

86. Muchos otros factores, además del crecimiento poblacional y la mavor densidad pobla. cional, evidentemente juegan un papel en este proceso. Por ejemplo, la incertidumbre respecto de los derechos de propiedad que es común a la explotación en pequeña escala desalienta los tipos permanentes de cultivos. 
87.C.E. Senbert y otros, "Effects on Land Clearing Methods and Soil Properties of an Ultisal and Crop Performance in the Amazon Jungle of Peru", Tropical Agriculture 54: 4 (Octubre 1977) 307-321.

88. Debe señalarse que las planes de colonización en el Perú no se han limitado a la Amazonía. Ver Craig L, Dogier, Land Development and Colonization in Latin America. Case Studies of Peru, Bolivia and Mexico (N. York, 1969), pp. 18-113.

89.De todos los migrantes de por vida registrados en el Censo del Perú de 1972, 7.2 \% se habian radicado en la Selva. (Ministerio de Trabajo. Dirección General de Empleo, Industrialización, Empleo y Migraciones Internas en el Perú), Tomo I (Lima, 1978), p. 80. No se sabe cuántos llegarán a la Selva como consecuencia de una colonización planificada, pero mi cálculo es una fracción de uno por ciento.

90. Aramburú cita una tasa de deserción de 86 \% en 1975 para un proyecto de asenta. miento en el valle del Huallaga (Ceja de Selva Central) que funciona desde 1966 con auspicio gubernamental. Ver aspects of Agrarian Structure in Peru. Problems of Colonization in Amazonia. Departament of Land Economy (Cambridge, 1975). Mimeografiado.

91. Dentro de este grupo se pueden diferenciar varios tipos según los diferentes grados y maneras de intervención gubernamental Ver CENCIRA, Recomendaciones Generales para una Política de Colonización en la Selva (Lima, sin fecha). Mimeografiado, Pp. 85-88. Se distingue entre la colonización espontánea guiada, colonización semi-planificada, planificada y colonización bajo dirección militar. En ésta última, las consideraciones geopolíticas prevaliecen sobre las consideraciones económicas.

92. Ver por ejemplo el plan de Tournavista en Michael Nelson, The Development of Tropical Lands. Policy Issues in Latin America (Baltimore, 1973), pp. 129-131.

93. Héctor Martínez, Las Colonizaciones Selváticas Dirigidas en el Perú. Antecedentes, Ac. tualidad y Perspectivas. (Lima, 1976), p. 109.

94. Estas razones se han resumido principalmente de las publicaciones siguientes:

- Carlos E. Aramburú, Aspects of Agrarian Structure in Peru, Problems of Colonization in Amazonía (Cambridge, 1975) Mimeografiado. Es una concisa pero excelente visión panorámica de diversos problemas de la colonización y la he usado profusamente.

- Carlos E. Aramburú, Las Migraciones a las Zonas de Colonización en la Selva Peruana: Perspectivas y Avances.

Próximamente en INANDEP. Las Migraciones a la Area Rural en el Perú.

- CENCIRA, Diagnóstico Socioeconómico de la Colonización Tingo María-Tocache. Campanilla. 2 Volúmenes (Lima, 1974) Mimeografiado. Existen pocos estudios de casos de colonización planificada en el Perú. Este estudio es una brillante excepción y contiene información e instrucciones sumamente interesantes. Desafortunadamente, sólo se publicaron 50 copias de este estudio y por eso es dificilmente accesible.

- Héctor Martínez, Las Colonizaciones Selváticas Diriqdas en el Perú, Antecedentes, Actualidad y Perspectivas (Lima 1976). Hasta la fecha, esta publicación es el primer y único intento de presentar una visión global y sistemática de la colonización planificada en la Selva Peruana. Contiene mucha información interesante y buena parte de las páginas siguientes se basa en dicho estudio.

- Román Robles Mendoxa, "Asentamientos Campesinos en la Colonización Tingo María-Tocache o los Mitimaes de Nuestros Tiempos", Discusión Antropológica, e:3 (1978): 103-115, El trabajo contiene informacion interesante pero hubiera ganado en profundidad y poder persuasivo si el estudio de caso fuera más sistemático y menos emotivo. Este documento critica con razón los planes de colonización como sus. títutos de reformas agrícolas pero se hace simplista al afin más que en el Perú rural no hay presión poblacional y que hay en realidad una escasez de mano de obra. La lectura de un artículo como "La Reforma y Más Allá: Del Fracaso del Modelo Agrario del Régimen Militar - Un Análisis Social y Político" por José María Caballero, "Crítica Andina" 2 (1978) debe convencer al autor de lo contrario.

- Roger Rumrrill y Pierre De Zutter, Amazonía y Capitalismo Los Condenados de la Selva (Lima, 1976), pp. 131-140. Este libro, básicamente del bando prudencialista, está escrito por dos periodistas. Ocasionalmente contiene información útil pero frecuentemente es superficial, por varias razones. Su objetivo de denuncia podría haber mejorado sustancialmente si estuviera sustentado por periodismo investiga- 
tivo de alta calidad. Desafortunadamente, el libro nunca trata un asunto a profundidad y rara vez menciona adecuadamente las fuentes de información. Abunda también en explicaciones y recomendaciones simplistas.

- Norman R. Stewart, "Some Problems in the development of Agricultural colonization in the Andean Oriente", Pxofessional Geographer 20 (1968): 33-38.

- R.F. Watters, La Agricultura Migratoria en Rerú (Mérida, Venezuela, 1968). Mimeografiado. Contiene también información esporádica pero interesante sobre los planes de colonización en el Perú. En las páginas siguientes, las referencias mencionadas se repetirín sólo si se indica especialmente. Los escritos de Marc Dourojeanni arriba mencionados contienen tambien referencias reiteradas a los efectos predominantemente desastrosos de la colonización sobre la calidad del suelo y la ecología del medio ambiente tropical. Para una extensa bibliografía sobre colonización y migra ción a la Selva ver Héctor Martínez, Migraciones internas en el Perú. Aproximación y Bibliografía (Lima, 1979). Manuscrito a publicarse en 1980 por el Instituto de Es tudios Peruanos. La Bibliografía sugiere que una altísima proporción de la literatura sobre el tema existe sólo en forma mimeografiada.

95.R.F. Watters, La Agricultura Migratoria en el Perú. (Mérida, Venezuela, 1968). Mimeografiado. También contiene información esporádica pero interesante sobre la coloniza. ción.

96. Héctor Martínez, Las Colonizaciones Selváticas Dirigidas en el Peru. Antecedentes, Ac. tualidad y Perspectivas (Lima, 1976) pp. 65-78.

97. Una visión global y concisa del proceso de adjudicación en una colonia puede encontrarse en CENCIRA, Diagnóstico Socioeconómico de la Colonización Tingo María-TocacheCampanilla, Primera parte (Lima, sin fecha). Mimeografiado, Pp. 134-147.

98. Román Robles Mendoza, "Asentamientos Campesinos en la Colonización Tingo María. Tocache o 'Los Mitimaes de Nuestros Tiempos'". Discusión Antropológica 3:3 (1978): 108.

99.R.F. Watters, La Agricultura Migratoria en el Perú. (Mérida, Venezuela, 1968). Mimeografiado. La p. 40 contiene también información esporádica pero interesante sobre colonización.

100. Héctor Martínez. Las Colonizaciones Selváticas Dirigidas en el Perú. Antecedentes, Actualidad y Perspectivas (Lima, 1976) pp. 75-77, p. 80.

101. Varios miembros de cooperativas agrarias de la Selva simplemente se han ido por falta de infraestructura, Ver Jorge Jelicio, La Reforma Asraria y la Ganaderra Lechera en el Perú. (Lima, 1978). p. 71.

102. CENCIRA, Diagnóstico Socioeconómico de la Colonización Tíngo María-TocacheCampanilla. Primera Parte (Lima, 1974). Mimeografiado, p. 157.

103. Héctor Martínez, Las Colonizaciones Selváticas Diridas en el Perú. Antecedentom, Actualidad y Perspectivas (Lima, 1976) p. 124.

104. CENCIRA, Diagnóstico Socioeconómico de la Colonización Tingo María-TocacheCampanilla. Segunda Parte (Lima, 1974). Mimeografiado, pp. 213-260.

105. CENCIRA, Recomendaciones generales para una política de colonización en la Selva (Lima, 1974).

CENCIRA (Centro Nacional de Capacitación e Investigación para la Reforma Agraria) es la rama de investigación y capacitación del Ministerio de Agricultura.

Este conjunto de recomendaciones se formuló sobre la base de cínco estudios de caso de planes de colonización existentes.

106. CENCIRA, Recomendaciones generales para una política de colonización en la Selva (Lima, 1974), P. 9.

107. CENCIRA, Recomendaciones generales para una política de colonización en la Selva (Lima, 1974), p. 10.

108. La colonización espontánea y planificada no son los únicos tipos de migración a la Selva. También hay un importante movimiento pendular entre la sierra y la Ceja de Selva que aparentemente data del tiempo preincaico (Ver por ejemplo la famosa teoría del control vertical en los niveles ecológicos en la economía de las sociedades andinas en John V. Murra, Formaeiones Económiess y Polítlces del Mundo Andino (Lima, 1975), pp. 59-115. Sin embargo, según una investigación inédita de Aramburú, por lo menos 
en el Departamento de Puno, este proceso parece menos importante que lo que se supone. Además para los planes de colonización debe hacerse una diatinción entre aquellas que involucran a colonos de una región diferente como los numerosos proyectos con campesinos serranos en la Ceja de Selva, y a los que se hacen con nativas de la Selva misma (frecuentemente en la parte de la llanura selvática).

Estos útimos planes, según Héctor Martínez, Las Colonizaciones Selviticas Durdoldan er ol Perú. Antecedentes, Actualidad y Porspectvas (Lima, 1976), p. 184, pueden llamarse más propiamente programas de reubicación rural. Ver también CENCIRA, Recomendaciones generales para una Política de colonización en la Selva (Lima, 1974), pp. 93-94.

109. Parz una descripción de un caso aparentemente exitoso de colonización ver Héctor Martínez, Las Mitaciones Altiplínicas y la Colonización de Tambopata (Lima, 1969). Aquí también la lentitud del gobierno para regularizar los títulos de tierras crea insegu. ridad. Es interesante señalar como en este caso de migración espontánea la estructura de clases típica del Altiplano sufrió varios cambios.

110. Ver R.F. Watters, L. Afrcultura Mitratori en Rerú, (Mérida, Venezuela, 1968) Mimeografiado, p. 33, que da cifras de crecimiento poblacional para los departamentos de la Selva en el período 1940-1961, y Carlos Aramburú "Las Migraciones a las zonas de Colonización en la Selva Peruana: Perspectivas y Avances", Debates en Aratropologra, 4 (1979): $81-94$ que da cifras de crecimiento poblacional del período $1950-1980$ para la Selva (rural y urbana) comparado con 12 Costa y la Sierra. El mismo artículo menciona tasas netas de migración para las mismas regiones que muestran que la tasa neta de migración para la Selva, aunque es menor que la de la costa, ha crecido más rápidamente entre 1940 y 1961 para la Selva que para la Costa.

El artículo contiene algunos datos y consideraciones muy interesantes sobre la migración a la Selva en comparación con la Costa. Aramburú actualmente está haciendo un estudio sobre todos los tipos de migración relativos al Departamento de Puno, El estudio se centra en las interrelaciones de los diversos cipos de migración dentro y desde el Departamento de Puno hacia la Costa y la Selva. Ver Organismo Regional de Desarrollo de Puno. Oficina Regional de Planificación, Diseño de la Investigación sobre "Migraciones y Colonización en Puno" (Puno, 1979). Mímeografiado.

111. Es interesante señalar que Hugo Blanco, uno de los principales lideres izquierdistas peruanos, explica la colonización espontánea en el pasado como consecuencia de la expansión del sistema de haciendas en la Sierra y del crecimiento poblacional (Hugo Blanco, Tierre o Muerte, Las Luchas Campesinas en Peru. (México, 1974), p. 16.

112. Ver R.F. Watters, La Agreultura Migratoria en Porú (Mérida, Venezuela, 1968) Mimeografiado, p. 33, pp. 35 y 37.

113. CENCIRA, Diagnóstico Socioeconómico de le Colonización Tingo María-TocacheCampanilla. Primera Parte (Lima, 1974). Mimeografiado p. 23.

114. Carlos Aramburú, "Las Migraciones a las zonas de Colonización en la Selva Peruana: Perspectivas y Avances" Debates en Aatropolocia, 4 (1979): 92.

116. CENCIRA, Diagnóstico Socioeconómico de la Colonización Tingo María- TocacheCampanilla. Primera Parte (Lima, 1974) Mimeografiado, Pp. 298-299.

116. Recomendaciones grupo "C". Tema: La Producción de Alimentos y la Problemática Rural. Reunión Nacional sobre Población. ('Tarma, junio 1979). Mimeografiado p. 21.

117. Luis J. Pax, La producción de Alimentos y la Problemítice Social, Reunín Nactonal sobre Población (Tarma, 1979). Mimeografiado p. 21.

118. Ver por ejemplo Marc J. Dourojeanni, Denarrollo Rural Integral en la Amazonía Peruana, con especial referencia a las actividades forestales. Primer Seminario sobre Alimentación y Agricultura en el Perú (Chaclacayo, 1979) Mimeografiado.

119. Por ejemplo desde octubre de 1979 se cobran tasas de interés más favorables para proyectos agrícolas o industriales. Ver El Comercio, setiembre 1979.

120. Constitución del Perú. Título IV del Régimen Económico - Capítulo II de los Recursos Naturales.

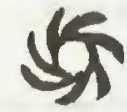

